\title{
The minimal density of triangles in tripartite graphs
}

\author{
Rahil Baber, J. Robert Johnson and John Talbot
}

\begin{abstract}
We determine the minimal density of triangles in a tripartite graph with prescribed edge densities. This extends a previous result of Bondy, Shen, Thomassé and Thomassen characterizing those edge densities guaranteeing the existence of a triangle in a tripartite graph. To be precise we show that a suitably weighted copy of the graph formed by deleting a certain 9-cycle from $K_{3,3,3}$ has minimal triangle density among all weighted tripartite graphs with prescribed edge densities. Supplementary materials are available with this article.
\end{abstract}

\section{Introduction}

Extremal questions for triangles in graphs have a very long history. The first such result, Mantel's theorem [6], tells us that a graph with $n$ vertices and more than $n^{2} / 4$ edges must contain at least one triangle.

For graphs with more than $n^{2} / 4$ edges it is natural to pose a quantitative question: what is the minimum number of triangles in a graph with a given number of edges? In this direction Razborov [7] determined (asymptotically) the minimal density of triangles in a graph of given edge density. This recent result was the cumulation of decades of contributions on this question due to Bollobás [1], Erdős [3], Lovász and Simonovits [5] and Fisher [4].

Recently, Bondy et al. [2] considered the very natural question of when a tripartite graph with prescribed edge densities must contain a triangle. (A tripartite graph is a graph $G=(V, E)$ for which there exists a partition of its vertices into three vertex classes such that all edges go between classes. The edge density between a pair of vertex classes $X, Y$ is simply the proportion of edges present between the two classes: $|E(X, Y)| /|X||Y|$.)

Bondy et al. characterized those triples of edge densities guaranteeing a triangle in a tripartite graph. As a special case they showed that any tripartite graph in which the density of edges between each pair of classes is greater than $1 / \varphi=0.618 \ldots$ contains a triangle (a precise statement of their full result can be found in the next section).

The aim of this paper is to prove a quantitative result which extends the theorem of Bondy et al. in the same way that Razborov's result extends Mantel's theorem.

The remainder of the paper is organized as follows. Formal definitions and main results are given in the next section. Our main result splits into two rather different cases and the following two sections contain their proofs. We finish with some conjectures and open problems. The proof relies on a computer search. Details of how to obtain the $\mathrm{C}++$ code used for this search may be found in the appendix.

\section{Definitions and results}

A tripartite graph is a graph $G=(V, E)$ for which there exists a partition of its vertices into three independent sets. Throughout, whenever we consider a tripartite graph we will implicitly assume that a fixed tripartition $V=A \dot{\cup} B \cup \dot{\cup}$ is given.

Received 7 October 2009; revised 8 April 2010.

2000 Mathematics Subject Classification 05 C35.

The last author is a Royal Society University Research Fellow. 
A weighted tripartite graph $(G, w)$ is a tripartite graph $G=(V, E)$ together with a weighting $w: V \rightarrow[0,1]$ satisfying

$$
\sum_{a \in A} w(a)=\sum_{b \in B} w(b)=\sum_{c \in C} w(c)=1 .
$$

The weight of an edge $x y \in E(G)$ is $w(x y)=w(x) w(y)$. The edge densities of $(G, w)$ are

$$
\alpha(G, w)=\sum_{b c \in E(B, C)} w(b c), \quad \beta(G, w)=\sum_{a c \in E(A, C)} w(a c), \quad \gamma(G, w)=\sum_{a b \in E(A, B)} w(a b) .
$$

We denote the set of all weighted tripartite graphs by $\operatorname{Tri}$. For $\alpha, \beta, \gamma \in[0,1]$ we let $\operatorname{Tri}(\alpha, \beta, \gamma)$ denote the set of all weighted tripartite graphs with edge densities $\alpha(G, w)=\alpha, \beta(G, w)=\beta$, $\gamma(G, w)=\gamma$.

Let $(G, w) \in$ Tri. A triangle in $G$ is a set of three vertices, $a \in A, b \in B, c \in C$, such that $a b, a c, b c \in E(G)$. We denote the set of all triangles in $G$ by $T(G)$. The weight of a triangle $x y z \in T(G)$ is $w(x y z)=w(x) w(y) w(z)$. The triangle density of $(G, w) \in$ Tri is

$$
t(G, w)=\sum_{a b c \in T(G)} w(a b c) .
$$

Note that with the obvious definitions of edge and triangle densities for simple tripartite graphs any such graph can be converted into a weighted tripartite graph with the same edge and triangle densities by setting the vertex weights to be $1 /|A|, 1 /|B|, 1 /|C|$ for vertices in classes $A, B, C$, respectively.

Also, any weighted tripartite graph with rational weights can be converted into a simple tripartite graph with the same edge and triangle densities by taking a suitable blow-up. To be precise, choose an integer $n$ so that $n w(v)$ is an integer for all vertices $v$ and replace each vertex of weight $x$ with $n x$ new vertices. The new vertices are clones of the old in the sense that we join a pair of vertices in the new graph if and only if the pair of vertices they arise from are adjacent in the weighted graph.

We are interested in how small the triangle density of a weighted tripartite graph with prescribed edge densities can be. Formally we wish to determine the following function. For $\alpha, \beta, \gamma \in[0,1]$ let

$$
T_{\min }(\alpha, \beta, \gamma)=\min _{(G, w) \in \operatorname{Tr} i(\alpha, \beta, \gamma)} t(G, w)
$$

It is not difficult to believe that this function is well-defined; however, for completeness we sketch a proof of this fact. Since this makes use of results from much later in the paper we suggest the reader takes this on trust until they reach the relevant results. Given $0 \leqslant \alpha, \beta, \gamma \leqslant 1$, Lemma 4.1 implies that $\operatorname{Tr} \mathbf{i}(\alpha, \beta, \gamma) \neq \emptyset$. Now Lemma 4.9 implies that when attempting to minimize $t(G, w)$ over $\operatorname{Tri}(\alpha, \beta, \gamma)$ we may restrict our search to the finite subfamily consisting of tripartite graphs with at most three vertices per class. Finally note that for a single tripartite graph $G$ the problem of determining the minimum value of $t(G, w)$, subject to the edge densities of $(G, w)$ being $\alpha, \beta, \gamma$, is a minimization problem for a continuous function over a compact domain. Hence $T_{\min }(\alpha, \beta, \gamma)$ is well-defined.

The following simple lemma shows that solving this weighted problem will give an asymptotic answer to the question of how many triangles a simple (unweighted) tripartite graph with given edge densities must have.

Lemma 2.1. (i) If $G$ is a simple tripartite graph with edge densities $\alpha, \beta, \gamma$ then it has triangle density at least $T_{\min }(\alpha, \beta, \gamma)$.

(ii) For rational $\alpha, \beta, \gamma$, if $(H, w) \in \operatorname{Tr} \mathbf{i}(\alpha, \beta, \gamma)$ then for all $\epsilon>0$ there is a simple tripartite graph $G$ with edge densities $\alpha, \beta, \gamma$ and triangle density at most $t(H, w)+\epsilon$. 


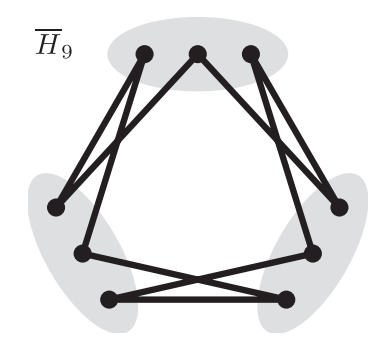

Figure 1. The tripartite complement of the graph $H_{9}$.

Proof. Part (i) is immediate since any tripartite graph can be transformed into a weighted tripartite graph by weighting vertices uniformly in each vertex class as described above.

For part (ii), let $w^{\prime}$ be a rational weighting of $H$ such that if the edge densities of $\left(H, w^{\prime}\right)$ are $\alpha^{\prime}, \beta^{\prime}, \gamma^{\prime}$ we have $\left|\alpha-\alpha^{\prime}\right|,\left|\beta-\beta^{\prime}\right|,\left|\gamma-\gamma^{\prime}\right|,\left|t(H, w)-t\left(H, w^{\prime}\right)\right|<\frac{1}{4} \epsilon$. We can do this since for a given $H$ the edge and triangle densities are continuous functions of the vertex weights. Now choose an integer $n$ so that $n w^{\prime}(v)$ is an integer for all vertices $v$, and $n^{2}\left|\alpha-\alpha^{\prime}\right|, n^{2} \mid \beta-$ $\beta^{\prime}\left|, n^{2}\right| \gamma-\gamma^{\prime} \mid$ are all integers. Blow up $H$ by replacing each vertex $v$ with $n w^{\prime}(v)$ cloned vertices to form a simple graph $G^{\prime}$ with $n$ vertices in each class. Finally, add or remove at most $\frac{3}{4} \epsilon n^{2}$ edges from $G^{\prime}$ to form a graph $G$ with edge densities $\alpha, \beta, \gamma$. This creates at most $\frac{3}{4} \epsilon n^{3}$ new triangles and so the triangle density of $G$ is at most $t\left(H, w^{\prime}\right)+\frac{3}{4} \epsilon<t(H, w)+\epsilon$.

Bondy et al. [2] proved the following sharp Turán-type result. If $(G, w) \in \operatorname{Tr} i(\alpha, \beta, \gamma)$ and $(\alpha, \beta, \gamma) \in R$, where

$$
R=\left\{(\alpha, \beta, \gamma) \in[0,1]^{3}: \alpha \beta+\gamma>1, \alpha \gamma+\beta>1, \beta \gamma+\alpha>1\right\},
$$

then $G$ must contain a triangle.

Theorem 2.2. $T_{\min }(\alpha, \beta, \gamma)=0 \Longleftrightarrow(\alpha, \beta, \gamma) \in[0,1]^{3} \backslash R$.

In particular, $T_{\min }(d, d, d)=0$ if and only if $d \leqslant 0.618 \ldots$ (the positive root of the quadratic $\left.x^{2}+x-1=0\right)$.

Our main result (Theorem 2.3) determines the minimal density of triangles in a weighted tripartite graph with prescribed edge densities.

The tripartite complement of a tripartite graph $G$ is the graph obtained by deleting the edges of $G$ from the complete tripartite graph on the same vertex classes as $G$. We will denote this by $\bar{G}$. Let $H_{9}$ be the graph whose tripartite complement is given in Figure 1.

Theorem 2.3. For any $(\alpha, \beta, \gamma) \in R$ there exists a weighting $w$ of $H_{9}$ such that $\left(H_{9}, w\right) \in$ $\operatorname{Tri}(\alpha, \beta, \gamma)$ and $t\left(H_{9}, w\right)=T_{\min }(\alpha, \beta, \gamma)$.

This theorem combined with Lemma 2.1 shows that a suitable blow-up of $H_{9}$ has asymptotically the minimum density of triangles for given edge densities.

There are two distinct cases to consider in the proof of Theorem 2.3, depending on the values of $\alpha, \beta, \gamma$. Let

$$
\Delta(\alpha, \beta, \gamma)=\alpha^{2}+\beta^{2}+\gamma^{2}-2 \alpha \beta-2 \alpha \gamma-2 \beta \gamma+4 \alpha \beta \gamma .
$$

We partition $R$ into two regions: $R_{1}$ and $R_{2}$ where

$$
R_{1}=\{(\alpha, \beta, \gamma) \in R: \Delta(\alpha, \beta, \gamma) \geqslant 0\}
$$

and $R_{2}=R \backslash R_{1}$. For $R_{1}$ we have the following result. 


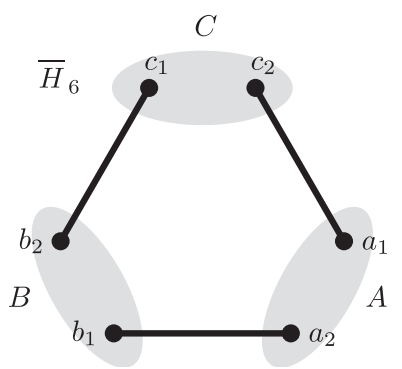

Figure 2. The tripartite complement of the graph $H_{6}$.
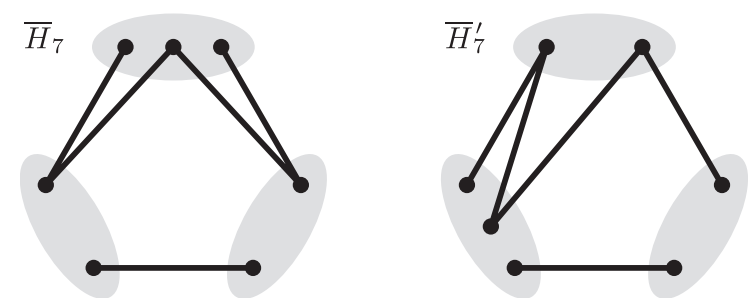

Figure 3. The tripartite complements of the graphs $H_{7}$ and $H_{7}^{\prime}$.

Theorem 2.4. If $(\alpha, \beta, \gamma) \in R_{1}$ and $H_{6}$ is the graph whose tripartite complement is given in Figure 2, then there exists a weighting $w$ such that $\left(H_{6}, w\right) \in \operatorname{Tr} \mathbf{i}(\alpha, \beta, \gamma)$, and for any such $w$

$$
T_{\min }(\alpha, \beta, \gamma)=t\left(H_{6}, w\right)=\alpha+\beta+\gamma-2
$$

Let $(G, w) \in \operatorname{Tr} \mathbf{i}(\alpha, \beta, \gamma)$. If $t(G, w)=T_{\min }(\alpha, \beta, \gamma)$ then $(G, w)$ is said to be extremal. If there does not exist $\left(G^{\prime}, w^{\prime}\right) \in \operatorname{Tr} \mathbf{T}(\alpha, \beta, \gamma)$ with $t\left(G^{\prime}, w^{\prime}\right)=t(G, w)$ and $\left|V\left(G^{\prime}\right)\right|<|V(G)|$, then $(G, w)$ is said to be vertex minimal. The tripartite graphs $G$ and $H$ with specified tripartitions are strongly-isomorphic if there is a graph isomorphism $f: G \rightarrow H$ such that the image of each vertex class in $G$ is a vertex class in $H$.

Theorem 2.5. If $(\alpha, \beta, \gamma) \in R_{2}$ and $(G, w) \in \operatorname{Tr} i(\alpha, \beta, \gamma)$ is extremal and vertex minimal, then $G$ is strongly-isomorphic to $H_{7}, H_{7}^{\prime}$ or $H_{9}$ (see Figure 3 ).

Proof of Theorem 2.3. The graphs $H_{6}, H_{7}$ and $H_{7}^{\prime}$ are induced subgraphs of $H_{9}$; hence Theorems 2.4 and 2.5 imply Theorem 2.3 .

We conjecture that in fact the extremal graph is always an appropriate weighting of $H_{7}$. This would also give a simple formula for $T_{\min }(\alpha, \beta, \gamma)$. See Section 5 for details.

3. Proof of Theorem 2.4 (the region $R_{1}$ )

Lemma 3.1. For any $\alpha, \beta, \gamma \in[0,1]$ and $(G, w) \in \operatorname{Tr} i(\alpha, \beta, \gamma)$ we have

$$
t(G, w) \geqslant \alpha+\beta+\gamma-2 \text {. }
$$

Proof. Define

$$
\mathbf{1}_{x y}=\left\{\begin{array}{ll}
1 & \text { if } x y \in E(G), \\
0 & \text { otherwise }
\end{array} \quad \mathbf{1}_{x y z}= \begin{cases}1 & \text { if } x y z \in T(G) \\
0 & \text { otherwise }\end{cases}\right.
$$


Given $a b c \in A \times B \times C$, the number of edges present between these three vertices is at most two unless $a b c$ forms a triangle. Hence

$$
\sum_{a b c \in A \times B \times C} w(a b c)\left(\mathbf{1}_{b c}+\mathbf{1}_{a c}+\mathbf{1}_{a b}\right) \leqslant \sum_{a b c \in A \times B \times C} w(a b c)\left(2+\mathbf{1}_{a b c}\right) .
$$

The left-hand side of (3.1) sums to $\alpha+\beta+\gamma$, while the right-hand side is $2+t(G, w)$. Therefore $t(G, w) \geqslant \alpha+\beta+\gamma-2$.

Lemma 3.2. If $w$ is a weighting of $H_{6}$ satisfying $\left(H_{6}, w\right) \in \operatorname{Tr} \mathbf{i}(\alpha, \beta, \gamma)$, then

$$
t\left(H_{6}, w\right)=\alpha+\beta+\gamma-2=T_{\min }(\alpha, \beta, \gamma) .
$$

For ease of notation the weight associated with a vertex is indicated with a hat above the label; for example, $w\left(b_{1}\right)$ is represented as $\hat{b}_{1}$.

Proof. Consider a general weighting of $H_{6}$ with vertices as labelled in Figure 2. We know $\hat{a}_{2}=1-\hat{a}_{1}, \hat{b}_{2}=1-\hat{b}_{1}$ and $\hat{c}_{2}=1-\hat{c}_{1}$ since the sum of the weights of the vertices in a class add up to one. Hence we can express the densities in terms of only $\hat{a}_{1}, \hat{b}_{1}$ and $\hat{c}_{1}$. The edge densities of $H_{6}$ are

$$
\alpha=1-\hat{c}_{1}+\hat{b}_{1} \hat{c}_{1}, \quad \beta=1-\hat{a}_{1}+\hat{a}_{1} \hat{c}_{1}, \quad \gamma=1-\hat{b}_{1}+\hat{a}_{1} \hat{b}_{1} .
$$

The triangle density is given by

$$
\begin{aligned}
t\left(H_{6}, w\right) & =\hat{a}_{1} \hat{b}_{1} \hat{c}_{1}+\left(1-\hat{a}_{1}\right)\left(1-\hat{b}_{1}\right)\left(1-\hat{c}_{1}\right) \\
& =1-\hat{a}_{1}-\hat{b}_{1}-\hat{c}_{1}+\hat{a}_{1} \hat{b}_{1}+\hat{a}_{1} \hat{c}_{1}+\hat{b}_{1} \hat{c}_{1} \\
& =\alpha+\beta+\gamma-2 .
\end{aligned}
$$

By Lemma 3.1 we have $t\left(H_{6}, w\right)=T_{\min }(\alpha, \beta, \gamma)$.

We now need to determine for which $(\alpha, \beta, \gamma) \in R$ a weighting $w$ exists such that $\left(H_{6}, w\right) \in$ $\operatorname{Tr} \mathbf{i}(\alpha, \beta, \gamma)$

LEMMA 3.3.

(i) If $(\alpha, \beta, \gamma) \in R$ then $\alpha, \beta, \gamma>0$.

(ii) If $(\alpha, \beta, \gamma) \in R_{2}$ then $0<\alpha, \beta, \gamma<1$.

Proof. If $(\alpha, \beta, \gamma) \in R$ and $\alpha=0$ then, since $\alpha \beta+\gamma>1$, we have $\gamma>1$, a contradiction. Similarly $\beta, \gamma>0$.

If $(\alpha, \beta, \gamma) \in R_{2}$ then $R_{2} \subseteq R$ implies that $\alpha, \beta, \gamma>0$. If $\alpha=1$ then $\Delta(\alpha, \beta, \gamma)=\Delta(1, \beta, \gamma)=$ $(1-\beta-\gamma)^{2} \geqslant 0$. But $(\alpha, \beta, \gamma) \in R_{2}$ implies that $\Delta(\alpha, \beta, \gamma)<0$, a contradiction. Similarly $\beta, \gamma<1$.

Lemma 3.4. For $(\alpha, \beta, \gamma) \in R$ there exists a weighting $w$ of $H_{6}$ such that $\left(H_{6}, w\right) \in$ $\operatorname{Tr} \mathbf{i}(\alpha, \beta, \gamma)$ if and only if $(\alpha, \beta, \gamma) \in R_{1}$.

Proof. If $(\alpha, \beta, \gamma) \in R$ then Lemma 3.3(i) implies that $\alpha, \beta, \gamma \neq 0$. First we will prove that if $(\alpha, \beta, \gamma) \in R$ and there exists a weighting $w$ such that $\left(H_{6}, w\right) \in \operatorname{Tr} i(\alpha, \beta, \gamma)$, then $(\alpha, \beta, \gamma) \in R_{1}$. 
Let us label the vertices of $H_{6}$ as in Figure 2. Suppose $w$ is the weighting of $H_{6}$ such that $\left(H_{6}, w\right) \in \operatorname{Tr} \mathbf{i}(\alpha, \beta, \gamma)$. The edge densities in terms of $\hat{a}_{1}, \hat{b}_{1}, \hat{c}_{1}$ are

$$
\begin{gathered}
\alpha=1-\hat{c}_{1}+\hat{b}_{1} \hat{c}_{1}, \\
\beta=1-\hat{a}_{1}+\hat{a}_{1} \hat{c}_{1}, \\
\gamma=1-\hat{b}_{1}+\hat{a}_{1} \hat{b}_{1} .
\end{gathered}
$$

Case 1: One of $\alpha, \beta, \gamma$ equals one. By Lemma 3.3(ii) $(\alpha, \beta, \gamma) \notin R_{2}$. Hence $(\alpha, \beta, \gamma) \in R$ implies $(\alpha, \beta, \gamma) \in R_{1}$.

Case 2: $\alpha, \beta, \gamma \neq 1$. Since $\alpha, \beta, \gamma \neq 1$ we have $\hat{a}_{1}, \hat{b}_{1}, \hat{c}_{1} \neq 0,1$. Rearranging (3.4) and (3.3) we can write $\hat{b}_{1}$ and $\hat{c}_{1}$ in terms of $\hat{a}_{1}$,

$$
\begin{gathered}
\hat{b}_{1}=\frac{1-\gamma}{1-\hat{a}_{1}}, \\
\hat{c}_{1}=\frac{\hat{a}_{1}+\beta-1}{\hat{a}_{1}} .
\end{gathered}
$$

Substituting into (3.2) and simplifying gives

$$
\alpha \hat{a}_{1}^{2}+(-\alpha+\beta-\gamma) \hat{a}_{1}+\gamma-\beta \gamma=0 .
$$

Hence

$$
\hat{a}_{1}=\frac{\alpha-\beta+\gamma \pm \sqrt{\Delta(\alpha, \beta, \gamma)}}{2 \alpha}
$$

and substituting back into (3.5) and (3.6) gives

$$
\begin{aligned}
& \hat{b}_{1}=\frac{\alpha+\beta-\gamma \pm \sqrt{\Delta(\alpha, \beta, \gamma)}}{2 \beta} \\
& \hat{c}_{1}=\frac{-\alpha+\beta+\gamma \pm \sqrt{\Delta(\alpha, \beta, \gamma)}}{2 \gamma}
\end{aligned}
$$

By the definition of a weighting we have $\hat{a}_{1}, \hat{b}_{1}, \hat{c}_{1} \in \mathbb{R} ;$ hence $\Delta(\alpha, \beta, \gamma) \geqslant 0$, and so $(\alpha, \beta, \gamma) \in$ $R_{1}$.

Next we will show that if $(\alpha, \beta, \gamma) \in R_{1}$ then there exists a weighting $w$ such that $\left(H_{6}, w\right) \in$ $\operatorname{Tri}(\alpha, \beta, \gamma)$.

Case 1: One of $\alpha, \beta, \gamma$ equals one. Without loss of generality, suppose $\alpha=1$. Since $(1, \beta, \gamma) \in R_{1} \subseteq R$ we have $\beta+\gamma>1$. It is easy to check that $\hat{a}_{1}=\gamma, \hat{b}_{1}=1, \hat{c}_{1}=(\beta+\gamma-1) / \gamma$ satisfy (3.2), (3.3), (3.4) and $\hat{a}_{1}, \hat{b}_{1}, \hat{c}_{1} \in[0,1]$ when $\beta+\gamma>1$. This is enough to define a weighting $w$ of $H_{6}$.

Case 2: $\alpha, \beta, \gamma \neq 1$. Since $\Delta(\alpha, \beta, \gamma) \geqslant 0$, we may define $\hat{a}_{1}, \hat{b}_{1}, \hat{c}_{1} \in \mathbb{R}$ by (3.7), (3.8) and (3.9), taking the positive square root in each case. Due to the way $\hat{a}_{1}, \hat{b}_{1}, \hat{c}_{1}$ were constructed they satisfy (3.2), (3.3) and (3.4). Hence if $\hat{a}_{1}, \hat{b}_{1}, \hat{c}_{1}$ form a weighting $w$ we will have $\left(H_{6}, w\right) \in \operatorname{Tri}(\alpha, \beta, \gamma)$. We need only prove $\hat{a}_{1}, \hat{b}_{1}, \hat{c}_{1} \in(0,1)$.

We will prove $\hat{a}_{1} \in(0,1)$; the proofs of $\hat{b}_{1}, \hat{c}_{1} \in(0,1)$ follow similarly. If $0<\alpha-\beta+\gamma$ then $0<\hat{a}_{1}$ because $\hat{a}_{1}$ is the positive square root version of (3.7). Now $(\alpha, \beta, \gamma) \in R$ implies $0<\alpha \beta+\gamma-1<\alpha+\gamma-\beta$, and consequently $0<\hat{a}_{1}$. By (3.7) if $\sqrt{\Delta(\alpha, \beta, \gamma)}<\alpha+\beta-\gamma$ then $\hat{a}_{1}<1$. Again $(\alpha, \beta, \gamma) \in R$ implies that $0<\alpha \gamma+\beta-1<\alpha+\beta-\gamma$. Hence if we can show $\Delta(\alpha, \beta, \gamma)<(\alpha+\beta-\gamma)^{2}$ we will be done. Expanding and simplifying yields $0<4 \alpha \beta(1-\gamma)$ which is true because $\alpha, \beta, \gamma \in(0,1)$.

Proof of Theorem 2.4. The result follows immediately from Lemmas 3.2 and 3.4. 


\section{Proof of Theorem 2.5 (the region $R_{2}$ )}

We will begin by introducing a new type of graph in $\S 4.1$ which will allow us to develop a series of conditions that extremal vertex minimal examples must satisfy. In $\S 4.2$ we outline an algorithm that allows us to utilize the results of $\S 4.1$ to search for the extremal vertex minimal graphs in a finite time. This algorithm produces 14 possible graphs. In $\S 4.3$ we eliminate those not strongly-isomorphic to $H_{7}, H_{7}^{\prime}$ and $H_{9}$ by analysing each of them in turn.

\subsection{Properties}

Our proof strategy for Theorem 2.5 is to establish various properties any extremal and vertex minimal weighted tripartite graph must satisfy. To prove these properties we introduce a new type of tripartite graph.

A doubly-weighted tripartite graph $(G, w, p)$ is a weighted tripartite graph $(G, w) \in$ Tri together with a function $p: E(G) \rightarrow(0,1]$. We denote the set of all doubly-weighted tripartite graphs by DTri. If $(G, w, p) \in \mathbf{D T r i}$ then the weight of an edge $x y \in E(G)$ is defined to be

$$
\lambda(x y)=w(x y) p(x y) .
$$

The edge density between a pair of vertex classes $X$ and $Y$ is

$$
\sum_{x y \in E(X, Y)} \lambda(x y)
$$

The triangle density is defined as

$$
t(G, w, p)=\sum_{a b c \in T(G)} p(a b) p(a c) p(b c) w(a b c) .
$$

Any $(G, w) \in$ Tri may be converted into a doubly-weighted tripartite graph $(G, w, p)$ with the same triangle and edge densities by adding the function $p: E(G) \rightarrow(0,1], p(e)=1$ for all $e \in E(G)$. Our next result allows us to do the reverse and convert a doubly-weighted tripartite graph into a weighted tripartite graph, leaving triangle and edge densities unchanged.

Lemma 4.1. Given $(G, w, p) \in \mathbf{D T r i}$ there exists $\left(G^{\prime}, w^{\prime}\right) \in$ Tri with the same triangle and edge densities.

For $(G, w, p) \in \mathbf{D T r i}$ we will say that $e \in E(G)$ is a partial edge if $p(e)<1$. To prove Lemma 4.1 we need a process to eliminate partial edges without affecting any of the densities.

For a graph $G$ and vertex $v \in V(G)$ let $\Gamma^{G}(v)$ denote the neighbourhood of $v$ in $G$. When no confusion can arise we write this simply as $\Gamma(v)$. Given a tripartite graph $G$ with a vertex class $X$ and $v \in V(G)$ we write $\Gamma_{X}^{G}(v)=\Gamma^{G}(v) \cap X$. Again when no confusion can arise we write this simply as $\Gamma_{X}(v)$.

Algorithm 1 Split. The algorithm Split takes as input $(G, w, p) \in$ DTri and an ordered pair of vertices $(x, y)$, such that $x y$ is a partial edge. Its output, Split $(G, w, p, x, y)$, is a doubly-weighted tripartite graph, which no longer contains the partial edge $x y$. If $\left(G^{\prime}, w^{\prime}, p^{\prime}\right)=$ Split $(G, w, p, x, y)$ then $G^{\prime}, w^{\prime}, p^{\prime}$ are formed as follows.

(i) Construct $G^{\prime}$ from $G$ by replacing the vertex $x$ by two new vertices $x_{0}$ and $x_{1}$ that lie in the same vertex class as $x$. Add edges from $x_{0}, x_{1}$ so that $\Gamma^{G^{\prime}}\left(x_{0}\right)=\Gamma^{G}(x) \backslash\{y\}$ and $\Gamma^{G^{\prime}}\left(x_{1}\right)=\Gamma^{G}(x)$.

(ii) Set $w^{\prime}\left(x_{0}\right)=w(x)(1-p(x y))$ and $w^{\prime}\left(x_{1}\right)=w(x) p(x y)$. Let $w^{\prime}(v)=w(v)$ for all $v \in$ $V(G) \backslash\{x\}$.

(iii) Set $p^{\prime}\left(x_{0} v\right)=p^{\prime}\left(x_{1} v\right)=p(x v)$ for all $v \in \Gamma^{G}(x) \backslash\{y\}$, and $p^{\prime}\left(x_{1} y\right)=1$. Let $p^{\prime}(u v)=p(u v)$ for all $u v \in E(G)$ such that $u, v \neq x$. 

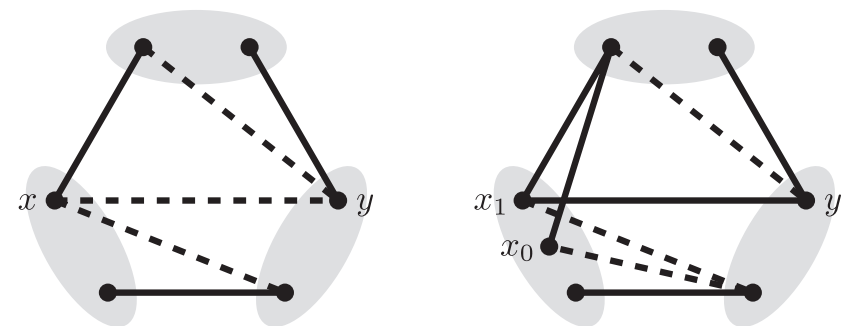

Figure 4. An example of $(G, w, p)$ and $\operatorname{Split}(G, w, p, x, y)$. Partial edges are represented by dotted lines and the solid lines are edges which $p$ maps to 1.

Note that in $\operatorname{Split}(G, w, p, y, x)$ (the result of applying Split to $(G, w, p)$ and $(y, x))$ the vertex $y$ would have been 'split' into two new vertices rather than $x$. It also does not contain the partial edge $x y$. So if we wish to remove the partial edge $x y$ we can choose between $\operatorname{Split}(G, w, p, x, y)$ and $\operatorname{Split}(G, w, p, y, x)$.

Figure 4 shows an example application of Split with 'before' and 'after' pictures of $(G, w, p)$ and $\operatorname{Split}(G, w, p, x, y)$.

Lemma 4.2. For any $(G, w, p) \in \mathbf{D T r i}$ and $x y$ a partial edge, $\left(G^{\prime}, w^{\prime}, p^{\prime}\right)=\operatorname{Split}(G, w, p$, $x, y)$ has the same triangle and edge densities as $(G, w, p)$.

Proof. Without loss of generality, let us assume $x \in A$ and $y \in B$. We will prove the result by calculating the difference in densities between $\left(G^{\prime}, w^{\prime}, p^{\prime}\right)$ and $(G, w, p)$ and showing them to be zero. The change in the edge density between classes $A$ and $B$ is

$$
\begin{aligned}
& w(y)\left(w^{\prime}\left(x_{1}\right) p^{\prime}\left(x_{1} y\right)-w(x) p(x y)\right) \\
& +\sum_{v \in \Gamma_{B}^{G}(x) \backslash\{y\}} w(v)\left(w^{\prime}\left(x_{0}\right) p^{\prime}\left(x_{0} v\right)+w^{\prime}\left(x_{1}\right) p^{\prime}\left(x_{1} v\right)-w(x) p(x v)\right)
\end{aligned}
$$

which is zero. Similarly the change in density between classes $A$ and $C$ is zero. There is no change in the density between classes $B$ and $C$ since the algorithm Split leaves this part of the graph untouched. The change in the triangle density is

$$
\begin{aligned}
& \sum_{x u v \in T(G), u \in B \backslash\{y\}, v \in C}\left(w^{\prime}\left(x_{0}\right)+w^{\prime}\left(x_{1}\right)-w(x)\right) w(u) w(v) p(x u) p(x v) p(u v) \\
& +\sum_{x y v \in T(G), v \in C}\left(w^{\prime}\left(x_{1}\right) p^{\prime}\left(x_{1} y\right)-w(x) p(x y)\right) w(y) w(v) p(x v) p(y v),
\end{aligned}
$$

which is zero; hence the triangle and edge densities do not change.

Proof of Lemma 4.1. Given $(G, w, p) \in \mathbf{D T r i}$, if $p(e)=1$ for all $e \in E(G)$ then the weighted tripartite graph $(G, w)$ will have the same densities as the doubly-weighted tripartite graph.

Suppose $(G, w, p)$ contains a partial edge $a v$, with $a \in A$. We can remove this partial edge by replacing $(G, w, p)$ by $\operatorname{Split}(G, w, p, a, v)$. Unfortunately, this may introduce new partial edges. However, we can show that by repeated applications of Split we will eventually remove all partial edges. Consider

$$
Z(G, w, p)=\sum_{v \in A} 3^{d_{z}(v)}
$$

where

$$
d_{z}(v)=|\{u \in V(G): u v \in E(G), p(u v) \neq 1\}|
$$


If $\left(G^{\prime}, w^{\prime}, p^{\prime}\right)=\operatorname{Split}(G, w, p, a, v)$ then $Z\left(G^{\prime}, w^{\prime}, p^{\prime}\right)<Z(G, w, p)$. This is because Split replaces vertex $a$ with the vertices $a_{0}$ and $a_{1}$, and so $Z$ changes by

$$
\begin{aligned}
3^{d_{z}\left(a_{0}\right)}+3^{d_{z}\left(a_{1}\right)}-3^{d_{z}(a)} & =3^{d_{z}(a)-1}+3^{d_{z}(a)-1}-3^{d_{z}(a)} \\
& =-3^{d_{z}(a)-1} .
\end{aligned}
$$

Since $Z$ is integral and is bounded below (by zero for instance), repeatedly applying Split will eventually remove all partial edges incident with $A$. Note that doing this will not have created any new partial edges between classes $B$ and $C$.

We can repeat this process on the partial edges leaving $B$, to get rid of the remaining partial edges. Let us call the resulting doubly-weighted tripartite graph $\left(G^{\prime \prime}, w^{\prime \prime}, p^{\prime \prime}\right)$. Since we created $\left(G^{\prime \prime}, w^{\prime \prime}, p^{\prime \prime}\right)$ only by applying Split, by Lemma 4.2, $(G, w, p)$ and $\left(G^{\prime \prime}, w^{\prime \prime}, p^{\prime \prime}\right)$ must have the same edge and triangle densities. Since $\left(G^{\prime \prime}, w^{\prime \prime}, p^{\prime \prime}\right)$ has no partial edges, $p^{\prime \prime}(e)=1$ for all $e \in E\left(G^{\prime \prime}\right)$; consequently $\left(G^{\prime \prime}, w^{\prime \prime}\right)$ has the same edge and triangle densities as $\left(G^{\prime \prime}, w^{\prime \prime}, p^{\prime \prime}\right)$ and therefore $(G, w, p)$.

Since we can convert easily between weighted and doubly-weighted tripartite graphs, it is useful to know when there exist doubly-weighted tripartite graphs with the same edge densities but with smaller triangle densities. Let $(G, w, p)$ be a doubly-weighted tripartite graph. By carefully modifying $p$ we can adjust the weights of edges whilst not affecting the edge densities and potentially decreasing the triangle density. Our next result lists a series of conditions under which this could occur.

Let $G$ be a tripartite graph with vertex classes $A, B, C$. For $a \in A, b \in B$ define

$$
C_{a b}=\{c \in C: a c, b c \in E(G)\} .
$$

Lemma 4.3. If $(G, w, p) \in \mathbf{D T r i}$ satisfies conditions (i)-(iv) below, then there exists $\left(G^{\prime}, w, p^{\prime}\right) \in \mathbf{D T r i}$ with the same edge densities as $(G, w, p)$ but $t\left(G^{\prime}, w, p^{\prime}\right)<t(G, w, p)$ :

(i) $w(v)>0$ for all $v \in V(G)$;

(ii) $p(e)=1$ for all $e \in E(A, C) \cup E(B, C)$;

(iii) there exist, not necessarily distinct, vertices $a_{0}, a_{1} \in A, b_{0}, b_{1} \in B$ such that $a_{1} b_{1} \in E(G)$ and either $a_{0} b_{0} \notin E(G)$ or $p\left(a_{0} b_{0}\right)<1$;

(iv) $\sum_{c \in C_{a_{0} b_{0}}} w(c)<\sum_{c \in C_{a_{1} b_{1}}} w(c)$.

Corollary 4.4. Let $(G, w) \in$ Tri. If there exist, not necessarily distinct, vertices $a_{0}, a_{1} \in$ $A, b_{0}, b_{1} \in B$ such that $a_{0} b_{0} \notin E(G), a_{1} b_{1} \in E(G)$ and $C_{a_{0} b_{0}}$ is a proper subset of $C_{a_{1} b_{1}}$, then $(G, w)$ is either not extremal or not vertex minimal.

Proof of Corollary 4.4. We will prove that if $(G, w)$ is vertex minimal then it is not extremal by applying Lemma 4.3.

Let $(G, w)$ be vertex minimal, so $w(v)>0$ for all $v \in V(G)$. We can add the function $p$ which maps all edges of $G$ to 1 to create $(G, w, p) \in$ DTri. Now $(G, w, p)$ has the same triangle and edge densities as $(G, w)$. By Lemma 4.1 it is enough to show that there exists $\left(G^{\prime}, w^{\prime}, p^{\prime}\right) \in \mathbf{D T r i}$ with the same edge densities as $(G, w, p)$ but a smaller density of triangles. Note that conditions (i)-(iii) in the statement of Lemma 4.3 hold for $(G, w, p)$. Thus Lemma 4.3 will provide such a $\left(G^{\prime}, w^{\prime}, p^{\prime}\right)$ if we can show that

$$
\sum_{c \in C_{a_{0} b_{0}}} w(c)<\sum_{c \in C_{a_{1} b_{1}}} w(c)
$$

Let $u \in C_{a_{1} b_{1}} \backslash C_{a_{0} b_{0}}$. Since $(G, w)$ is vertex minimal, $w(u)>0$. Hence

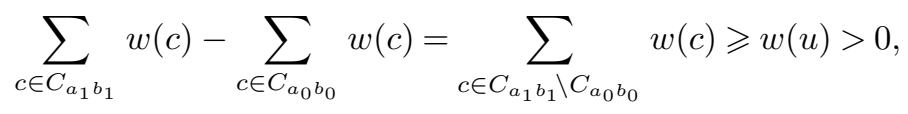

in which case all the conditions of Lemma 4.3 are satisfied, and $(G, w)$ is not extremal. 


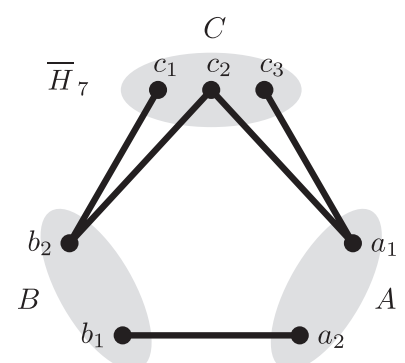

Figure 5. The tripartite complement of the graph $H_{7}$.

Proof of Lemma 4.3. If $a_{0} b_{0} \notin E(G)$ let $G^{\prime}$ be the graph produced from $G$ by adding the edge $a_{0} b_{0}$. If $a_{0} b_{0} \in E(G)$ then let $G^{\prime}=G$. Define $p^{\prime}: E\left(G^{\prime}\right) \rightarrow(0,1]$ by $p^{\prime}(e)=p(e)$ for $e \in E\left(G^{\prime}\right) \backslash\left\{a_{0} b_{0}, a_{1} b_{1}\right\}$ and

$$
\begin{aligned}
p^{\prime}\left(a_{0} b_{0}\right)= \begin{cases}\frac{\delta}{w\left(a_{0}\right) w\left(b_{0}\right)} & \text { if } a_{0} b_{0} \notin E(G), \\
p\left(a_{0} b_{0}\right)+\frac{\delta}{w\left(a_{0}\right) w\left(b_{0}\right)} & \text { if } a_{0} b_{0} \in E(G),\end{cases} \\
p^{\prime}\left(a_{1} b_{1}\right)=p\left(a_{1} b_{1}\right)-\frac{\delta}{w\left(a_{1}\right) w\left(b_{1}\right)},
\end{aligned}
$$

where $\delta>0$ is chosen sufficiently small so that $p^{\prime}\left(a_{0} b_{0}\right), p^{\prime}\left(a_{1} b_{1}\right) \in(0,1)$.

The weights and edges have not changed between classes $A, C$ and $B, C$. Consequently, the corresponding edge densities will be the same in $(G, w, p)$ and $\left(G^{\prime}, w, p^{\prime}\right)$. However, the edge density between class $A$ and $B$, and the triangle densities may have changed. The difference in the $A, B$ edge density between $\left(G^{\prime}, w, p^{\prime}\right)$ and $(G, w, p)$ is

$$
w\left(a_{0}\right) w\left(b_{0}\right) \frac{\delta}{w\left(a_{0}\right) w\left(b_{0}\right)}-w\left(a_{1}\right) w\left(b_{1}\right) \frac{\delta}{w\left(a_{1}\right) w\left(b_{1}\right)}=0 .
$$

The change in triangle density is

$$
\sum_{a_{0} b_{0} c \in T\left(G^{\prime}\right)} w\left(a_{0}\right) w\left(b_{0}\right) w(c) \frac{\delta}{w\left(a_{0}\right) w\left(b_{0}\right)}-\sum_{a_{1} b_{1} c \in T\left(G^{\prime}\right)} w\left(a_{1}\right) w\left(b_{1}\right) w(c) \frac{\delta}{w\left(a_{1}\right) w\left(b_{1}\right)}
$$

which simplifies to

$$
\delta\left(\sum_{a_{0} b_{0} c \in T\left(G^{\prime}\right)} w(c)-\sum_{a_{1} b_{1} c \in T\left(G^{\prime}\right)} w(c)\right)<0,
$$

where the final inequality follows from condition (iv).

Hence the density of triangles in $\left(G^{\prime}, w, p^{\prime}\right)$ is smaller than that in $(G, w, p)$, but the edge densities are the same in both.

Lemma 4.5. Consider the graph $H_{7}$ whose tripartite complement is given in Figure 5. If $(\alpha, \beta, \gamma) \in R_{2}$ then there exists a weighting $w$ such that $\left(H_{7}, w\right) \in \operatorname{Tr} \mathbf{T}(\alpha, \beta, \gamma)$ and $t\left(H_{7}, w\right)=$ $2 \sqrt{\alpha \beta(1-\gamma)}+2 \gamma-2$. Furthermore, $t\left(H_{7}, w\right) \leqslant t\left(H_{7}, w^{\prime}\right)$ for all weightings $w^{\prime}$, such that $\left(H_{7}, w^{\prime}\right) \in \operatorname{Tr} \mathbf{i}(\alpha, \beta, \gamma)$.

Proof. If $(\alpha, \beta, \gamma) \in R_{2}$ then, by Lemma 3.3(ii), we know that $0<\alpha, \beta, \gamma<1$. Consider a general weighting of $H_{7}$, with vertices labelled as in Figure 5. If such a weighting of $H_{7}$ has edge densities $\alpha, \beta, \gamma$ then $\alpha, \beta, \gamma<1$ implies that $\hat{a}_{1} \neq 0,1, \gamma$. Now given $\alpha, \beta, \gamma$ and $\hat{a}_{1} \neq 0,1, \gamma$ we have enough information to deduce the rest of the weights of the vertices. (Note that this 
may not be an actual weighting since some of these values may lie outside of $[0,1]$.)

$$
\begin{array}{lll}
\hat{a}_{2}=1-\hat{a}_{1}, & \hat{b}_{1}=\frac{1-\gamma}{1-\hat{a}_{1}}, & \hat{b}_{2}=\frac{\gamma-\hat{a}_{1}}{1-\hat{a}_{1}}, \\
\hat{c}_{1}=1-\frac{1-\beta}{\hat{a}_{1}}, & \hat{c}_{3}=1-\frac{(1-\alpha)\left(1-\hat{a}_{1}\right)}{\gamma-\hat{a}_{1}}, & \\
\hat{c}_{2}=\frac{1-\beta}{\hat{a}_{1}}+\frac{(1-\alpha)\left(1-\hat{a}_{1}\right)}{\gamma-\hat{a}_{1}}-1, & &
\end{array}
$$

which have been deduced from

$$
\begin{array}{cll}
1=\hat{a}_{1}+\hat{a}_{2}, & 1-\gamma=\hat{a}_{2} \hat{b}_{1}, & 1=\hat{b}_{1}+\hat{b}_{2}, \\
1-\beta=\left(1-\hat{c}_{1}\right) \hat{a}_{1}, & 1-\alpha=\left(1-\hat{c}_{3}\right) \hat{b}_{2}, & 1=\hat{c}_{1}+\hat{c}_{2}+\hat{c}_{3},
\end{array}
$$

respectively. There are two triangles in $H_{7}$, with weights $\hat{a}_{1} \hat{b}_{1} \hat{c}_{1}$ and $\hat{a}_{2} \hat{b}_{2} \hat{c}_{3}$; hence the triangle density is

$$
\hat{a}_{1}\left(\frac{1-\gamma}{1-\hat{a}_{1}}\right)\left(1-\frac{1-\beta}{\hat{a}_{1}}\right)+\left(1-\hat{a}_{1}\right)\left(\frac{\gamma-\hat{a}_{1}}{1-\hat{a}_{1}}\right)\left(1-\frac{(1-\alpha)\left(1-\hat{a}_{1}\right)}{\gamma-\hat{a}_{1}}\right)
$$

which simplifies to

$$
2 \gamma-2+\frac{\beta(1-\gamma)}{1-\hat{a}_{1}}+\alpha\left(1-\hat{a}_{1}\right)
$$

This expression is minimized when $1-\hat{a}_{1}=\sqrt{\beta(1-\gamma) / \alpha}$, and consequently we obtain the desired triangle density of $2 \sqrt{\alpha \beta(1-\gamma)}+2 \gamma-2$. We must now show that the vertex weights implied by $\hat{a}_{1}=1-\sqrt{\beta(1-\gamma) / \alpha}$ all lie in $[0,1]$ and that $\hat{a}_{1} \neq \gamma, 0,1$. Since the sum of the weights in each class equals one, in order to show that all weights lie in $[0,1]$ it is sufficient to show that they are all non-negative.

If $\hat{a}_{1}=\gamma$ then $1-\gamma=\sqrt{\beta(1-\gamma) / \alpha}$, which rearranges to $\alpha \gamma+\beta-\alpha=0$. However, $\alpha \gamma+\beta-\alpha>\alpha \gamma+\beta-1>0\left(\right.$ as $\left.(\alpha, \beta, \gamma) \in R_{2} \subseteq R\right)$, and hence $\hat{a}_{1} \neq \gamma$. Moreover, $1-\hat{a}_{1}$ is clearly positive, proving that $0<\hat{a}_{2}$ and $\hat{a}_{1} \neq 1$. Showing $0<\hat{a}_{1}$ is equivalent to proving $\sqrt{\beta(1-\gamma) / \alpha}<1$ which is true if $0<\beta \gamma+\alpha-\beta$, and this holds because $\beta \gamma+\alpha-\beta>\beta \gamma+$ $\alpha-1>0$. Since $\hat{b}_{2}$ equals $1-\sqrt{\alpha(1-\gamma) / \beta}$, a similar argument shows that $\hat{b}_{1}, \hat{b}_{2}>0$. It is also straightforward to show that $\hat{c}_{1}, \hat{c}_{3}>0$, but showing $\hat{c}_{2}>0$ requires more work. Using $\hat{c}_{1}+\hat{c}_{2}+\hat{c}_{3}=1, \hat{c}_{1}=1-(1-\beta) / \hat{a}_{1}$ and $\hat{c}_{3}=1-(1-\alpha) / \hat{b}_{2}$ we obtain

Hence $\hat{c}_{2}>0$ if and only if

$$
\hat{c}_{2}=-1+\frac{(1-\beta) \hat{b}_{2}+(1-\alpha) \hat{a}_{1}}{\hat{a}_{1} \hat{b}_{2}} \text {. }
$$

$$
\hat{a}_{1} \hat{b}_{2}<(1-\beta) \hat{b}_{2}+(1-\alpha) \hat{a}_{1} .
$$

Substituting $\hat{a}_{1}=1-\sqrt{\beta(1-\gamma) / \alpha}$ and $\hat{b}_{2}=1-\sqrt{\alpha(1-\gamma) / \beta}$ yields

$$
\alpha+\beta-\gamma<2 \sqrt{\alpha \beta(1-\gamma)}
$$

Now $\alpha+\beta-\gamma>\alpha \gamma+\beta-1>0$; hence $0<\hat{c}_{2}$ if and only if $(\alpha+\beta-\gamma)^{2}<4 \alpha \beta(1-\gamma)$. Collecting all the terms onto the left-hand side shows that we require $\Delta(\alpha, \beta, \gamma)<0$, which we have from the fact that $(\alpha, \beta, \gamma) \in R_{2}$.

Lemma 4.6. For any $(\alpha, \beta, \gamma) \in R_{2}$,

$$
T_{\min }(\alpha, \beta, \gamma)<\min \{\alpha \beta+\gamma-1, \alpha \gamma+\beta-1, \beta \gamma+\alpha-1\} .
$$

Proof of Lemma 4.6. Without loss of generality, let us assume that

$$
\alpha \beta+\gamma-1=\min \{\alpha \beta+\gamma-1, \alpha \gamma+\beta-1, \beta \gamma+\alpha-1\} .
$$



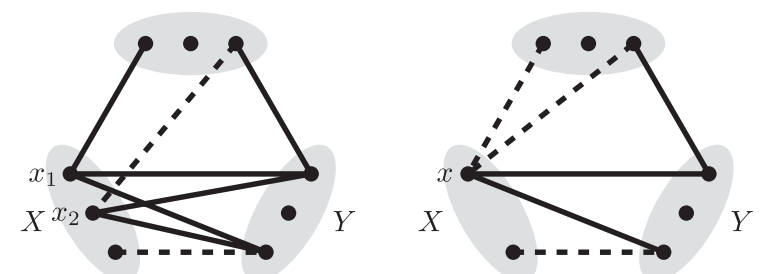

Figure 6. An example of $(G, w, p)$ and $\operatorname{Merge}\left(G, w, p, x_{1}, x_{2}\right)$. Partial edges are represented by dotted lines and the solid lines are edges which $p$ maps to 1.

By Lemma 4.5 we know that for any $(\alpha, \beta, \gamma) \in R_{2}$ there exists a weighting $w$ such that $\left(H_{7}, w\right) \in \operatorname{Tri}(\alpha, \beta, \gamma)$ and $t\left(H_{7}, w\right)=2 \sqrt{\alpha \beta(1-\gamma)}+2 \gamma-2$. Hence $T_{\min }(\alpha, \beta, \gamma) \leqslant$ $2 \sqrt{\alpha \beta(1-\gamma)}+2 \gamma-2$. If $\alpha \beta+\gamma-1 \leqslant 2 \sqrt{\alpha \beta(1-\gamma)}+2 \gamma-2$ then

$$
\alpha \beta+1-\gamma \leqslant 2 \sqrt{\alpha \beta(1-\gamma)} \text {. }
$$

Squaring and rearranging yields

$$
(\alpha \beta+\gamma-1)^{2} \leqslant 0 .
$$

Since $(\alpha, \beta, \gamma) \in R_{2} \subseteq R$ we know $\alpha \beta+\gamma-1>0$ holds true; hence we have a contradiction.

Lemma 4.7. Let $(\alpha, \beta, \gamma) \in R_{2}$. If $(G, w) \in \operatorname{Tr} \mathbf{i}(\alpha, \beta, \gamma)$ is extremal, then $|A|,|B|,|C| \geqslant 2$.

To prove Lemma 4.7 we will require the following algorithm.

Algorithm 2 Merge. The algorithm Merge takes as input $(G, w, p) \in \mathbf{D T r i}$, and two distinct vertices $x_{1}, x_{2} \in X$, where $X$ is one of the vertex classes of $G$. The vertices $x_{1}, x_{2}$ must satisfy, for some vertex class $Y \neq X$, the properties $\Gamma_{Y}\left(x_{1}\right)=\Gamma_{Y}\left(x_{2}\right), w\left(x_{1}\right)+$ $w\left(x_{2}\right)>0$ and $p\left(x_{1} y\right)=p\left(x_{2} y\right)=1$ for all $y \in \Gamma_{Y}\left(x_{1}\right)$. The output of the algorithm is represented by $\operatorname{Merge}\left(G, w, p, x_{1}, x_{2}\right)$ and is a doubly-weighted tripartite graph in which $x_{1}, x_{2}$ have been replaced by a single new vertex $x$. For convenience let us write $\left(G^{\prime}, w^{\prime}, p^{\prime}\right)=$ $\operatorname{Merge}\left(G, w, p, x_{1}, x_{2}\right)$. Now $G^{\prime}, w^{\prime}, p^{\prime}$ are formed as follows.

(i) Construct $G^{\prime}$ from $G$ by replacing the vertices $x_{1}, x_{2}$ by a new vertex $x$ in $X$. Add edges from $x$ so that $\Gamma^{G^{\prime}}(x)=\Gamma^{G}\left(x_{1}\right) \cup \Gamma^{G}\left(x_{2}\right)$.

(ii) Set $w^{\prime}(x)=w\left(x_{1}\right)+w\left(x_{2}\right)$. Let $w^{\prime}(v)=w(v)$ for all $v \in V\left(G^{\prime}\right) \backslash\{x\}$.

(iii) For $u, v \in V\left(G^{\prime}\right) \backslash\{x\}$ and $u v \in E\left(G^{\prime}\right)$, let $p^{\prime}(u v)=p(u v)$. For $x v \in E\left(G^{\prime}\right)$ set

$$
p^{\prime}(x v)= \begin{cases}w\left(x_{1}\right) p\left(x_{1} v\right) / w^{\prime}(x) & \text { if } x_{1} v \in E(G), x_{2} v \notin E(G), \\ w\left(x_{2}\right) p\left(x_{2} v\right) / w^{\prime}(x) & \text { if } x_{1} v \notin E(G), x_{2} v \in E(G), \\ \left(w\left(x_{1}\right) p\left(x_{1} v\right)+w\left(x_{2}\right) p\left(x_{2} v\right)\right) / w^{\prime}(x) & \text { if } x_{1} v \in E(G), x_{2} v \in E(G) .\end{cases}
$$

Observe that for $y \in Y$ we have $x y \in E\left(G^{\prime}\right)$ if and only if $x_{1} y, x_{2} y \in E(G)$ and in this case $p(x y)=1$. It is easy to check that the edge and triangle densities of $(G, w, p)$ and $\left(G^{\prime}, w^{\prime}, p^{\prime}\right)$ are the same.

Proof of Lemma 4.7. Suppose $(G, w)$ is extremal and, without loss of generality, vertex class $C=\{c\}$ contains exactly one vertex. We can assume $w(v) \neq 0$ for all $v \in V(G)$, as any vertices with weight zero can be removed without affecting any of the densities. Create a doubly-weighted tripartite graph $(G, w, p)$ with the same densities as $(G, w)$ by setting $p(e)=1$ for all $e \in E(G)$. We will show that the triangle density of $(G, w, p)$ is at least $\alpha \beta+\gamma-1$ and consequently, by Lemma $4.6,(G, w)$ is not extremal. 


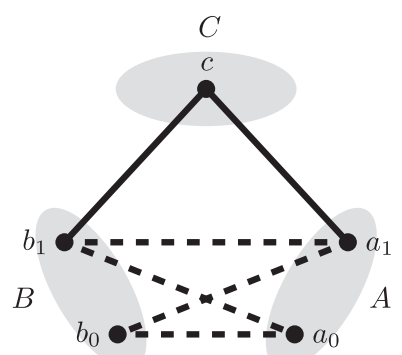

Figure 7. A graph with $|C|=1$ after merging vertices in $A$ and $B$. The dotted lines represent edges that may or may not be in the graph.

Since $(\alpha, \beta, \gamma) \in R_{2}$, by Lemma 3.3(ii) we have $\beta \neq 0,1$. Moreover, since $C=\{c\}$ we know that there must exist a vertex in $A$ whose neighbourhood in $C$ is empty and another whose neighbourhood in $C$ is $\{c\}$. We can replace all vertices $a \in A$ satisfying $\Gamma_{C}(a)=\emptyset$ by a single vertex $a_{0}$ via repeated applications of the Merge algorithm on pairs of such vertices. Similarly we can replace all vertices with $\Gamma_{C}(a)=\{c\}$ by a single vertex $a_{1}$. Having done this we obtain a doubly-weighted graph in which $A=\left\{a_{0}, a_{1}\right\}, a_{1} c$ is an edge and $a_{0} c$ is a non-edge. Note the edges and weights between $B$ and $C$ remain unchanged but we may have modified the edges and weights between $A$ and $B$.

By a similar argument we can reduce $B$ to two vertices $b_{0}, b_{1}$, with $b_{1} c$ an edge and $b_{0} c$ a non-edge. Let us call this doubly-weighted graph $\left(G^{\prime}, w^{\prime}, p^{\prime}\right)$, and note it has the same densities as $(G, w, p)$ and hence $(G, w)$. By construction we have

$$
a_{0} c, b_{0} c \notin E\left(G^{\prime}\right), \quad a_{1} c, b_{1} c \in E\left(G^{\prime}\right), \quad p^{\prime}\left(a_{1} c\right)=p^{\prime}\left(b_{1} c\right)=1
$$

(see Figure 7).

We now have enough information to determine the weights of all of the vertices:

$$
w^{\prime}(c)=1, \quad w^{\prime}\left(a_{1}\right)=\beta, \quad w^{\prime}\left(a_{0}\right)=1-\beta, \quad w^{\prime}\left(b_{1}\right)=\alpha, \quad w^{\prime}\left(b_{0}\right)=1-\alpha .
$$

The only information we are lacking about $\left(G^{\prime}, w^{\prime}, p^{\prime}\right)$ is which edges are present in $E(A, B)$ and what their weights are. However, since $(\alpha, \beta, \gamma) \in R$, Theorem 2.2 implies that $G^{\prime}$ contains a triangle. Hence $a_{1} b_{1} \in E(A, B)$. Since $C_{a_{1} b_{1}}=\{c\}$ and $C_{a_{0} b_{0}}=C_{a_{0} b_{1}}=C_{a_{1} b_{0}}=\emptyset$, Lemma 4.3 tells us that $(G, w)$ will not be extremal unless $a_{0} b_{0}, a_{0} b_{1}, a_{1} b_{0}$ are all edges which $p^{\prime}$ maps to 1 .

Now, $a_{1} b_{1} c$ is the only triangle in the doubly-weighted tripartite graph; hence the triangle density is $w^{\prime}\left(a_{1}\right) w^{\prime}\left(b_{1}\right) p^{\prime}\left(a_{1} b_{1}\right)=\lambda\left(a_{1} b_{1}\right)$ (as $w^{\prime}(c), p^{\prime}\left(a_{1} c\right), p^{\prime}\left(b_{1} c\right)$ are all 1$)$. By the definition of edge density in a doubly-weighted tripartite graph, we have

$$
\begin{aligned}
\gamma & =\lambda\left(a_{0} b_{0}\right)+\lambda\left(a_{0} b_{1}\right)+\lambda\left(a_{1} b_{0}\right)+\lambda\left(a_{1} b_{1}\right) \\
& =(1-\alpha)(1-\beta)+\alpha(1-\beta)+(1-\alpha) \beta+t\left(G^{\prime}, w^{\prime}, p^{\prime}\right) \\
& =1-\alpha \beta+t\left(G^{\prime}, w^{\prime}, p^{\prime}\right) .
\end{aligned}
$$

Hence the triangle density is $\alpha \beta+\gamma-1$, which by Lemmas 4.6 and 4.1 implies that $(G, w)$ is not extremal.

Lemma 4.8. If $(\alpha, \beta, \gamma) \in R_{2},(G, w) \in \operatorname{Tr} \mathbf{i}(\alpha, \beta, \gamma)$ and, for all $a_{1}, a_{2} \in A, \Gamma_{C}\left(a_{1}\right)=$ $\Gamma_{C}\left(a_{2}\right)$, then $(G, w)$ is not extremal.

Proof. If there exist any vertices with weight zero, we can remove them without affecting the densities. Convert the resulting weighted tripartite graph into a doubly-weighted tripartite 
graph and reduce $A$ down to a single vertex, by repeated applications of Merge on the vertices in $A$. Any partial edges that appear will lie in $E(A, B)$.

Now repeatedly apply Split choosing to replace vertices in $B$ rather than $A$, until no more partial edges remain. Consequently we have modified the weighted graph into a new weighted graph with the same densities and now $|A|=1$. By Lemma 4.7 we know this is not extremal and hence $(G, w)$ was not extremal.

Our next lemma is an adaptation of a convexity argument by Bondy et al. (see [2, proof of Theorem 3]). This allows us to reduce the problem of determining which tripartite graphs can be both vertex minimal and extremal to those with at most three vertices in each vertex class.

Lemma 4.9. If $(G, w) \in$ Tri is extremal and vertex minimal, then $|A|,|B|,|C| \leqslant 3$.

Again we introduce an algorithm to prove this lemma.

Algorithm 3 Reduce. The algorithm Reduce takes as input $(G, w) \in$ Tri and a vertex class $X$ of $G$, satisfying $|X|>3$. Its output, represented by $\operatorname{Reduce}(G, w, X)$, is a weighted tripartite graph, which has the same edge densities as $(G, w)$, but with $|X| \leqslant 3$, and a triangle density at most that of $(G, w)$.

To help explain the algorithm we will suppose $X=A$ (the other choices of $X$ work similarly). For each vertex $a_{i} \in A$ let

$$
\beta_{i}=\sum_{c \in \Gamma_{C}\left(a_{i}\right)} w(c), \quad \gamma_{i}=\sum_{b \in \Gamma_{B}\left(a_{i}\right)} w(b), \quad t_{i}=\sum_{b c \in E(B, C), a_{i} b c \in T(G)} w(b c) .
$$

By definition

$$
\beta=\sum_{i=1}^{|A|} w\left(a_{i}\right) \beta_{i}, \quad \gamma=\sum_{i=1}^{|A|} w\left(a_{i}\right) \gamma_{i}, \quad t(G, w)=\sum_{i=1}^{|A|} w\left(a_{i}\right) t_{i} .
$$

Consider the convex hull

$$
P=\left\{\sum_{i=1}^{|A|} x_{i}\left(\beta_{i}, \gamma_{i}, t_{i}\right): \sum_{i=1}^{|A|} x_{i}=1 \text { and } x_{i} \geqslant 0\right\} .
$$

Setting $x_{i}=w\left(a_{i}\right)$ shows that $(\beta, \gamma, t(G, w))$ lies in $P$. By varying the values of the $x_{i}$ we can decrease the value of $t(G, w)$ to $t^{\prime}$ such that $\left(\beta, \gamma, t^{\prime}\right)$ lies on the boundary of $P$. Moreover, by triangulating the facet of $P$ containing $\left(\beta, \gamma, t^{\prime}\right)$, we can express $\left(\beta, \gamma, t^{\prime}\right)$ as a convex combination of at most three elements of $\left\{\left(\beta_{i}, \gamma_{i}, t_{i}\right): 1 \leqslant i \leqslant|A|\right\}$. Consequently we can write

$$
\left(\beta, \gamma, t^{\prime}\right)=\sum_{i=1}^{|A|} x_{i}\left(\beta_{i}, \gamma_{i}, t_{i}\right)
$$

where $\sum x_{i}=1$ and at most three of the $x_{i}$ are positive, the rest being zero. Now define a new weighting $w^{\prime}$ for $G$ by $w^{\prime}\left(a_{i}\right)=x_{i}, w^{\prime}(v)=w(v)$ for $v \in V(G) \backslash A$. The weighted tripartite graph $\left(G, w^{\prime}\right)$ has the same edge densities as $(G, w)$ and a new triangle density $t^{\prime}$ satisfying $t^{\prime} \leqslant t(G, w)$. Furthermore, we can remove the zero weighted vertices from $A$ so that $|A| \leqslant 3$ and the densities are unchanged.

Proof of Lemma 4.9. Suppose $(G, w)$ is extremal and vertex minimal with, without loss of generality, $|A|>3$. Now, using $\operatorname{Algorithm} 3$, $\operatorname{Reduce}(G, w, A)$ has the same densities as $(G, w)$ (since $(G, w)$ is extremal), but it has fewer vertices, contradicting the vertex minimality of $(G, w)$. 
Lemma 4.10. Let $(G, w)$ be a weighted tripartite graph. If there exist distinct vertices $a_{1}, a_{2} \in A$ with $\Gamma_{C}\left(a_{1}\right)=\Gamma_{C}\left(a_{2}\right)$ and $|B|=3$, then $(G, w)$ is not extremal or not vertex minimal.

Proof. Convert $(G, w)$ into a doubly-weighted tripartite graph and replace $a_{1}, a_{2}$ with a vertex $a$ by applying Merge (we may assume $w\left(a_{1}\right)+w\left(a_{2}\right)>0$ by vertex minimality of $(G, w)$ ). Now $A$ has reduced in size by one. If there are partial edges they will lie between classes $A$ and $B$. Use the Split algorithm to remove them, choosing to replace vertices in $B$ rather than $A$. Now convert the doubly-weighted graph back into a weighted graph. This weighted graph will have the same densities as $(G, w), A$ has one less vertex, and $|B| \geqslant 3$. If $|B|=3$ then this weighted graph is of smaller order than $(G, w)$. If $|B|>3$ we can use Reduce to modify the weights of vertices in $B$, such that at most three of them have a non-zero weight. Simply remove all vertices with zero weight and the resulting graph will be of smaller order than $G$, contradicting vertex minimality.

Lemma 4.11. Consider a weighted graph $(G, w)$. If there exist distinct vertices $a_{1}, a_{2} \in A$ with $\Gamma\left(a_{1}\right)=\Gamma\left(a_{2}\right)$ then $(G, w)$ is not vertex minimal.

Proof. Remove vertex $a_{2}$ and increase the weight of $a_{1}$ by $w\left(a_{2}\right)$. The resulting weighted graph has the same densities as $(G, w)$.

Lemma 4.12. Given a tripartite graph $G$ with $|A|=3$, not necessarily distinct, vertices $a_{0}, a_{1} \in A, b_{0}, b_{1} \in B$ such that $a_{0} b_{0} \notin E(G), a_{1} b_{1} \in E(G)$ and $C_{a_{0} b_{0}}=C_{a_{1} b_{1}}$, construct two graphs $G_{1}, G_{2}$ as follows.

(i) Let $G_{1}^{\prime}=G-a_{1} b_{1}$. Construct $G_{1}$ from $G_{1}^{\prime}$ by adding a new vertex $a_{2}$ to $A$ and adding edges incident to $a_{2}$ so that $\Gamma^{G_{1}}\left(a_{2}\right)=\Gamma^{G_{1}^{\prime}}\left(a_{0}\right) \cup\left\{b_{0}\right\}$.

(ii) Let $G_{2}^{\prime}=G+a_{0} b_{0}$. Construct $G_{2}$ from $G_{2}^{\prime}$ by adding a new vertex $a_{2}$ to $A$ and adding edges incident to $a_{2}$ so that $\Gamma^{G_{2}}\left(a_{2}\right)=\Gamma^{G_{2}^{\prime}}\left(a_{1}\right) \backslash\left\{b_{1}\right\}$.

Note that in $G_{1}$ and $G_{2}$ we have $|A|=4$. Let $\mathcal{H}$ denote the family of eight graphs constructed from $G_{1}$ or $G_{2}$ by deleting a single vertex from $A$.

If $(G, w)$ is extremal and vertex minimal then there exists $H \in \mathcal{H}$ and a weighting $w^{\prime}$ of $H$ such that $\left(H, w^{\prime}\right)$ has the same edge densities as $(G, w)$ and is also extremal and vertex minimal.

Proof. Our proof will involve first showing that there exists a weighting $w^{\prime \prime}$ of $G_{1}, G_{2}$ such that either $\left(G_{1}, w^{\prime \prime}\right)$ or $\left(G_{2}, w^{\prime \prime}\right)$ have the same densities as $(G, w)$.

Form a doubly-weighted graph $(G, w, p)$ with $p(e)=1$ for all $e \in E(G)$. Since $C_{a_{0} b_{0}}=C_{a_{1} b_{1}}$, if we add the edge $a_{0} b_{0}$ to $G$ we can move weight from edge $a_{1} b_{1}$ to $a_{0} b_{0}$, by modifying $p\left(a_{1} b_{1}\right)$ and $p\left(a_{0} b_{0}\right)$, whilst keeping the edge and triangle densities constant. If we move as much weight as we can from $a_{1} b_{1}$ to $a_{0} b_{0}$, one of two things must happen. Either we manage to make $p\left(a_{0} b_{0}\right)=1$ before $p\left(a_{1} b_{1}\right)$ reaches zero, or $p\left(a_{1} b_{1}\right)$ reaches zero (so we remove edge $a_{1} b_{1}$ ) and $p\left(a_{0} b_{0}\right) \leqslant 1$. In either case we have at most one partial edge, either $a_{1} b_{1}$ or $a_{0} b_{0}$. We can remove the partial edge by an application of the Split algorithm, introducing an extra vertex into class $A$. The two possible resulting graphs are $G_{2}$ and $G_{1}$, respectively. Hence there exists a weighting $w^{\prime \prime}$ such that either $\left(G_{1}, w^{\prime \prime}\right)$ or $\left(G_{2}, w^{\prime \prime}\right)$ have the same densities as $(G, w)$.

Without loss of generality, let us assume $\left(G_{1}, w^{\prime \prime}\right)$ has the same densities as $(G, w)$. Since $|A|=4$ for $G_{1}$, applying the Reduce algorithm will remove at least one vertex from $A$ to create a doubly-weighted graph, say $\left(H, w^{\prime}\right)$, with the same edge densities and possibly a smaller triangle density. However, since $t(G, w)=t\left(G_{1}, w^{\prime \prime}\right) \geqslant t\left(H, w^{\prime}\right)$ and $(G, w)$ is extremal, we must have $t(G, w)=t\left(H, w^{\prime}\right)$, implying $\left(H, w^{\prime}\right)$ is extremal. We can also conclude, by the vertex minimality of $(G, w)$, that $H$ is formed from $G_{1}$ by removing exactly one vertex from $A$. 

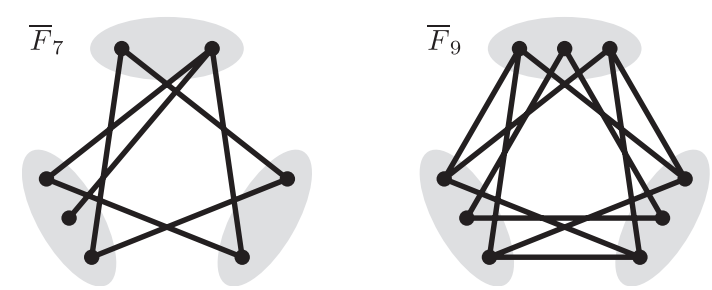

FIGURE 8. The tripartite complements of the graphs $F_{7}$ and $F_{9}$.

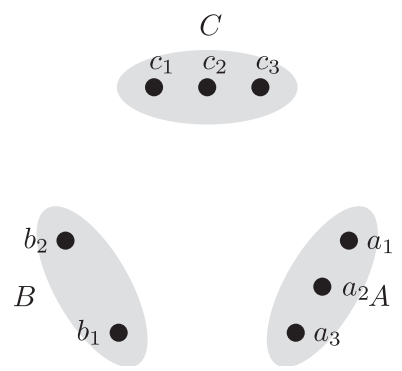

Figure 9. Canonical labelling of vertices and vertex classes.

\subsection{Search for extremal examples}

We have now developed a number of important conditions that any vertex minimal extremal examples must satisfy. These will, eventually, allow us to conduct an exhaustive search for such graphs (with the aid of a computer). This will then leave us with a small number of possible extremal graphs which we will deal with by hand.

Recall that the tripartite graphs $G$ and $H$ (as always with specified tripartitions) are stronglyisomorphic if there is a graph isomorphism $f: G \rightarrow H$ such that the image of each vertex class in $G$ is a vertex class in $H$.

It turns out that if we can eliminate graphs that are strongly-isomorphic to two particular examples, $F_{7}$ and $F_{9}$ (see Figure 8), then our computer search will be able to eliminate many more possible extremal vertex minimal examples, and thus reduce the amount of work we will finally need to do by hand.

For ease of notation we will henceforth implicitly label the vertices and vertex classes of all figures as in Figure 9. Indices of vertices start at 1 and increase clockwise. Recall that the weight associated with a vertex is indicated with a hat above the label; for example, $w\left(b_{1}\right)$ is represented as $\hat{b}_{1}$.

Lemma 4.13. If $(\alpha, \beta, \gamma) \in R_{2}$ then, for all weightings $w$ such that $\left(F_{7}, w\right) \in \operatorname{Tri}(\alpha, \beta, \gamma)$, $\left(F_{7}, w\right)$ is either not extremal or not vertex minimal.

To prove Lemma 4.13, we first need to prove the following result about the graph $F_{6}$ given in Figure 10.

Lemma 4.14. For any $\alpha, \beta, \gamma \in[0,1]$ and weighting $w$ satisfying $\left(F_{6}, w\right) \in \operatorname{Tri}(\alpha, \beta, \gamma)$ we have

$$
t\left(F_{6}, w\right) \geqslant \min \{\alpha \beta+\gamma-1, \alpha \gamma+\beta-1, \beta \gamma+\alpha-1\}
$$




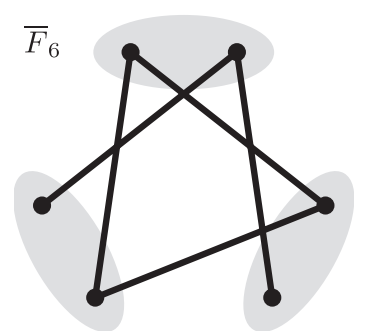

Figure 10. The tripartite complement of the graph $F_{6}$.

Proof. Suppose (4.1) fails to hold. Since $F_{6}$ contains only one triangle, $a_{2} b_{2} c_{1}$, and using the fact that $\hat{a}_{2}=1-\hat{a}_{1}, \hat{b}_{2}=1-\hat{b}_{1}, \hat{c}_{2}=1-\hat{c}_{1}$, we have

$$
\begin{gathered}
t\left(F_{6}, w\right)=\left(1-\hat{a}_{1}\right)\left(1-\hat{b}_{1}\right) \hat{c}_{1}, \\
\alpha=\hat{b}_{1}\left(1-\hat{c}_{1}\right)+\left(1-\hat{b}_{1}\right) \hat{c}_{1}, \\
\beta=\hat{a}_{1}\left(1-\hat{c}_{1}\right)+\left(1-\hat{a}_{1}\right) \hat{c}_{1}, \\
\gamma=1-\hat{a}_{1} \hat{b}_{1} .
\end{gathered}
$$

Substitute (4.2), (4.3), (4.4) and (4.5) into $t\left(F_{6}, w\right)<\alpha \beta+\gamma-1$ and rearrange to obtain

$$
\left(1-2 \hat{a}_{1}\right)\left(1-2 \hat{b}_{1}\right)\left(1-\hat{c}_{1}\right) \hat{c}_{1}+\hat{a}_{1} \hat{b}_{1} \hat{c}_{1}<0 .
$$

This implies (since $\hat{c}_{1}, 1-\hat{c}_{1}, \hat{a}_{1}, \hat{b}_{1} \geqslant 0$ ) that $0<1-2 \hat{a}_{1}$ or $0<1-2 \hat{b}_{1}$ (if $1-2 \hat{a}_{1} \leqslant 0$ and $1-2 \hat{b}_{1} \leqslant 0$ then the left-hand side of (4.6) would be non-negative).

If $0<1-2 \hat{a}_{1}$ is true then substitute (4.2), (4.3), (4.4) and (4.5) into $t\left(F_{6}, w\right)<\alpha \gamma+\beta-1$ and rearrange to obtain

$$
\hat{a}_{1} \hat{b}_{1} \hat{c}_{1}\left(2-\hat{b}_{1}\right)+\hat{a}_{1}\left(1-\hat{b}_{1}\right)^{2}\left(1-\hat{c}_{1}\right)+\left(1-2 \hat{a}_{1}\right)\left(1-\hat{b}_{1}\right)\left(1-\hat{c}_{1}\right)<0 .
$$

But each term on the left-hand side is strictly non-negative so we have a contradiction.

If instead $0<1-2 \hat{b}_{1}$ holds, then looking at $t\left(F_{6}, w\right)<\beta \gamma+\alpha-1$ yields

$$
\hat{a}_{1} \hat{b}_{1} \hat{c}_{1}\left(2-\hat{a}_{1}\right)+\hat{b}_{1}\left(1-\hat{a}_{1}\right)^{2}\left(1-\hat{c}_{1}\right)+\left(1-2 \hat{b}_{1}\right)\left(1-\hat{a}_{1}\right)\left(1-\hat{c}_{1}\right)<0,
$$

which is similarly false.

Proof of Lemma 4.13. Suppose $\left(F_{7}, w\right)$ is extremal and vertex minimal. We may assume $w(v) \in(0,1)$ for all $v \in V\left(F_{7}\right)$. If $t\left(F_{7}, w\right) \geqslant \alpha \beta+\gamma-1$ then, by Lemma $4.6,\left(F_{7}, w\right)$ is not extremal, so we may assume that

$$
t\left(F_{7}, w\right)<\alpha \beta+\gamma-1
$$

and similarly

$$
\begin{aligned}
& t\left(F_{7}, w\right)<\alpha \gamma+\beta-1 \\
& t\left(F_{7}, w\right)<\beta \gamma+\alpha-1
\end{aligned}
$$

Consider moving all the weight from $b_{3}$ to $b_{2}$ to create the following weighting $w^{\prime}$ of $F_{7}$ defined formally as $w^{\prime}(v)=w(v)$ for all $v \in V(G) \backslash\left\{b_{2}, b_{3}\right\}, w^{\prime}\left(b_{2}\right)=w\left(b_{2}\right)+w\left(b_{3}\right)$ and $w^{\prime}\left(b_{3}\right)=0$. Changing the weighting from $w$ to $w^{\prime}$ does not change the edge density between $A$ and $C$, or $B$ and $C$, but it may have increased the edge density between $A$ and $B$ and the triangle density. Let us call the new edge density, between $A$ and $B, \gamma^{\prime}$. Its value can be expressed in terms of the old weights and densities

$$
\gamma^{\prime}=\gamma+\hat{a}_{2} \hat{b}_{3}
$$


Similarly

$$
t\left(F_{7}, w^{\prime}\right)=t\left(F_{7}, w\right)+\hat{a}_{2} \hat{b}_{3} \hat{c}_{1}
$$

If we can show that

$$
\begin{aligned}
& t\left(F_{7}, w^{\prime}\right)<\alpha \beta+\gamma^{\prime}-1, \\
& t\left(F_{7}, w^{\prime}\right)<\alpha \gamma^{\prime}+\beta-1, \\
& t\left(F_{7}, w^{\prime}\right)<\beta \gamma^{\prime}+\alpha-1
\end{aligned}
$$

all hold, then, since $w^{\prime}\left(b_{3}\right)=0$, we could remove $b_{3}$ from $F_{7}$ leaving all densities unchanged, and the resulting graph would be strongly-isomorphic to $F_{6}$. This contradicts Lemma 4.14; hence our assumption that $\left(F_{7}, w\right)$ is extremal and vertex minimal must be false.

First let us show that (4.10) holds. Consider

$$
\begin{aligned}
\alpha \beta+\gamma^{\prime}-1-t\left(F_{7}, w^{\prime}\right) & =\alpha \beta+\left(\gamma+\hat{a}_{2} \hat{b}_{3}\right)-1-\left(t\left(F_{7}, w\right)+\hat{a}_{2} \hat{b}_{3} \hat{c}_{1}\right) \\
& =\alpha \beta+\gamma-1-t\left(F_{7}, w\right)+\hat{a}_{2} \hat{b}_{3}\left(1-\hat{c}_{1}\right) \\
& >0 .
\end{aligned}
$$

The inequality holds because $\alpha \beta+\gamma-1-t\left(F_{7}, w\right)>0$ by $(4.7)$ and $\hat{a}_{2}, \hat{b}_{3}, \hat{c}_{1} \in(0,1)$.

To prove (4.11) we look at

$$
\begin{aligned}
\alpha \gamma^{\prime}+\beta-1-t\left(F_{7}, w^{\prime}\right) & =\alpha\left(\gamma+\hat{a}_{2} \hat{b}_{3}\right)+\beta-1-\left(t\left(F_{7}, w\right)+\hat{a}_{2} \hat{b}_{3} \hat{c}_{1}\right) \\
& =\alpha \gamma+\beta-1-t\left(F_{7}, w\right)+\hat{a}_{2} \hat{b}_{3}\left(\alpha-\hat{c}_{1}\right) .
\end{aligned}
$$

We know $\alpha \gamma+\beta-1-t\left(F_{7}, w\right)>0$ by (4.8), and $\hat{a}_{2}, \hat{b}_{3}>0$, so all we have to do is show that $\alpha-\hat{c}_{1} \geqslant 0$. By definition $\alpha$ is the sum of the weighted edges between $B$ and $C$, and hence

$$
\begin{aligned}
\alpha & =\left(\hat{b}_{2}+\hat{b}_{3}\right) \hat{c}_{1}+\hat{b}_{1} \hat{c}_{2} \\
& =\left(1-\hat{b}_{1}\right) \hat{c}_{1}+\hat{b}_{1}\left(1-\hat{c}_{1}\right) .
\end{aligned}
$$

Therefore

$$
\begin{aligned}
\alpha-\hat{c}_{1} & =\left(1-\hat{b}_{1}\right) \hat{c}_{1}+\hat{b}_{1}\left(1-\hat{c}_{1}\right)-\hat{c}_{1} \\
& =\hat{b}_{1}\left(1-2 \hat{c}_{1}\right) .
\end{aligned}
$$

Since $\hat{b}_{1}$ is greater than zero, we require $\hat{c}_{1} \leqslant 1 / 2$.

Consider $C_{a_{1} b_{1}}=\left\{c_{2}\right\}$ and $C_{a_{2} b_{2}}=\left\{c_{1}\right\}$. Construct $\left(F_{7}, w, p\right) \in$ DTri by setting $p(e)=1$ for all edges of $F_{7}$. If $\hat{c}_{2}<\hat{c}_{1}$ then, by Lemma 4.3 , we know we can achieve a smaller triangle density. Therefore $\hat{c}_{1} \leqslant \hat{c}_{2}$ must hold, or equivalently $\hat{c}_{1} \leqslant 1 / 2\left(\right.$ as $\left.\hat{c}_{1}+\hat{c}_{2}=1\right)$.

Similarly, to prove (4.12) consider

$$
\beta \gamma^{\prime}+\alpha-1-t\left(F_{7}, w^{\prime}\right)=\beta \gamma+\alpha-1-t\left(F_{7}, w\right)+\hat{a}_{2} \hat{b}_{3}\left(\beta-\hat{c}_{1}\right) .
$$

By (4.9) we need only show $\beta-\hat{c}_{1} \geqslant 0$, which is true because $\beta-\hat{c}_{1}=\hat{a}_{1}\left(1-2 \hat{c}_{1}\right), \hat{a}_{1}>0$ and $\hat{c}_{1} \leqslant 1 / 2$.

Lemma 4.15. For all weightings $w$ such that $\left(F_{9}, w\right) \in \mathbf{T r i},\left(F_{9}, w\right)$ is either not extremal or not vertex minimal.

Proof. Let us assume that $\left(F_{9}, w\right)$ is extremal and vertex minimal, in which case $w(v) \neq 0$ for all $v \in V\left(F_{9}\right)$. Construct $\left(F_{10}, w^{\prime}\right) \in \operatorname{Tri}$ from $\left(F_{9}, w\right)$ as follows.

(i) Create $F_{10}$ from $F_{9}$ by removing the edge $a_{3} c_{1}$. Add a new vertex into $C$, labelled $c_{4}$, and add in edges so that $\Gamma^{F_{10}}\left(c_{4}\right)=\Gamma_{B}^{F_{9}}\left(c_{1}\right) \cup A$.

(ii) Set $w^{\prime}(v)=w(v)$ for all $v \in V\left(F_{10}\right) \backslash\left\{c_{1}, c_{4}\right\}$. Let

$$
w^{\prime}\left(c_{1}\right)=\frac{w\left(a_{1}\right) w\left(c_{1}\right)}{w\left(a_{1}\right)+w\left(a_{3}\right)} \quad \text { and } \quad w^{\prime}\left(c_{4}\right)=\frac{w\left(a_{3}\right) w\left(c_{1}\right)}{w\left(a_{1}\right)+w\left(a_{3}\right)} .
$$


The edge density between $A$ and $B$ remains unchanged and it is easy to check that the density between $B$ and $C$ also has not changed. The change in edge density between $A$ and $C$ is

$$
w\left(a_{2}\right) w^{\prime}\left(c_{1}\right)+w^{\prime}\left(c_{4}\right)-w\left(a_{2}\right) w\left(c_{1}\right)-w\left(a_{3}\right) w\left(c_{1}\right)=0 .
$$

The triangles in $F_{9}$ are $a_{1} b_{3} c_{2}, a_{2} b_{1} c_{3}, a_{3} b_{2} c_{1}$ and the triangles in $F_{10}$ are $a_{1} b_{3} c_{2}, a_{2} b_{1} c_{3}, a_{1} b_{2} c_{4}$, $a_{3} b_{2} c_{4}$. Hence the change in triangle density between $\left(F_{9}, w\right)$ and $\left(F_{10}, w^{\prime}\right)$ is

$$
\left(w\left(a_{1}\right)+w\left(a_{3}\right)\right) w\left(b_{2}\right) w^{\prime}\left(c_{4}\right)-w\left(a_{3}\right) w\left(b_{2}\right) w\left(c_{1}\right)=0 .
$$

Therefore $\left(F_{9}, w\right)$ and $\left(F_{10}, w^{\prime}\right)$ have the same triangle and edge densities.

Note that $\Gamma_{C}^{F_{10}}\left(a_{1}\right)=\Gamma_{C}^{F_{10}}\left(a_{3}\right)=\left\{c_{2}, c_{4}\right\}$. Since $|C|=4$ we can apply the Reduce algorithm to class $C$ in $F_{10}$, and the resultant output $\left(F^{\prime \prime}, w^{\prime \prime}\right) \in$ Tri has the same edge densities and the same triangle density (because $\left(F_{9}, w\right)$ is extremal). Moreover, $\left|V\left(F^{\prime \prime}\right)\right|=\left|V\left(F_{9}\right)\right|\left(\right.$ as $\left(F_{9}, w\right)$ is vertex minimal) and $\Gamma_{C}^{F^{\prime \prime}}\left(a_{1}\right)=\Gamma_{C}^{F^{\prime \prime}}\left(a_{3}\right)$. Hence we can apply Lemma 4.10 to $\left(F^{\prime \prime}, w^{\prime \prime}\right)$, showing that it is either not extremal or not vertex minimal and so the same must be true of $\left(F_{9}, w\right)$.

Our goal is to produce a list of all tripartite graphs $G$ for which there exists a weighting $w$ such that $(G, w) \in \operatorname{Tr} i(\alpha, \beta, \gamma)$ is extremal and vertex minimal for some $(\alpha, \beta, \gamma) \in R_{2}$. With this aim in mind we have developed a number of results that allow us to show $(G, w)$ is not extremal or not vertex minimal by simply examining $G$, irrespective of the weighting $w$.

By Lemmas 4.7 and 4.9 we need only consider tripartite graphs $G$ in which all vertex classes contain either two or three vertices. This reduces the problem to a finite search. However, tripartite graphs with $|A|=|B|=|C|=3$ can contain 27 possible edges, so naively there are at least $2^{27} \approx 100000000$ graphs to consider. We can decrease the possible number of graphs by looking at only those that contain triangles, since otherwise $(\alpha, \beta, \gamma) \notin R$ by Theorem 2.2. By Lemma 4.11 we know that if $G$ has a class containing a pair of vertices with identical neighbours then it is not vertex minimal (because we can move all the weight from one vertex to the other). Similarly the more technical results given in Corollary 4.4, Lemmas 4.8, 4.10, $4.12,4.13$ and 4.15 can also be used to eliminate graphs without knowledge of the vertex weights. Tripartite graphs that are strongly-isomorphic to graphs eliminated by these results will also not be extremal or not vertex minimal, and so may also be discarded.

Unfortunately applying Corollary 4.4, Lemmas 4.8, 4.10-4.13, 4.15 and Theorem 2.2 to over 100000000 tripartite graphs would take too long to perform by hand, but can easily be done by computer. A $\mathrm{C}++$ implementation is given in the Appendix (which is available via the multimedia link on the online article webpage). This algorithm produces a list of possible extremal vertex minimal tripartite graphs in $R_{2}$, which are equivalent up to strong-isomorphism to the 14 graphs given in Figure 11. To decrease the number further we will have to check each of these graphs by hand.

\subsection{Specific graphs}

To complete the proof of Theorem 2.5 we need to eliminate the 11 graphs found by the computer search, other than $H_{7}, H_{7}^{\prime}$ and $H_{9}$. (In the list of 14 graphs these are $G_{8}, G_{7}$ and $G_{13}$, respectively.)

To be precise we will show that for each $G_{i}, 1 \leqslant i \leqslant 14, i \neq 7,8,13$, if $(\alpha, \beta, \gamma) \in R_{2}$ then there does not exist a weighting $w$ such that $\left(G_{i}, w\right) \in \operatorname{Tr} \mathbf{i}(\alpha, \beta, \gamma)$ and $\left(G_{i}, w\right)$ is both extremal and vertex minimal.

Lemma 4.16. If $(\alpha, \beta, \gamma) \in R_{2}$ then, for all weightings $w$ such that $\left(G_{1}, w\right) \in \operatorname{Tr} \mathbf{i}(\alpha, \beta, \gamma)$, $\left(G_{1}, w\right)$ is not extremal.

Proof. $G_{1}$ is strongly-isomorphic to $F_{6}$. Hence Lemmas 4.14 and 4.6 imply $\left(G_{1}, w\right)$ is not extremal. 

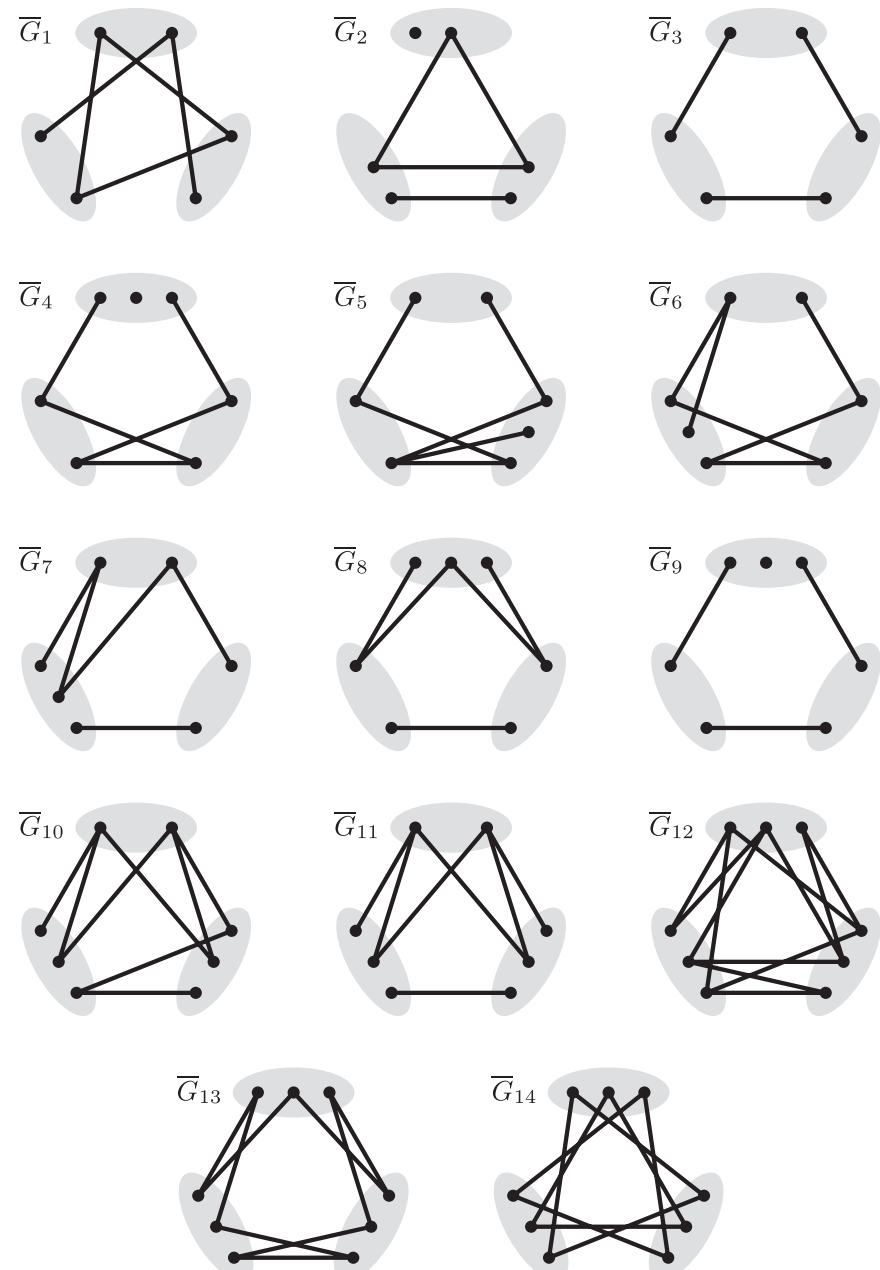

FiguRE 11. The tripartite complements of the graphs produced by the computer search.

Lemma 4.17. If $(\alpha, \beta, \gamma) \in R_{2}$ then, for all weightings $w$ such that $\left(G_{2}, w\right) \in \operatorname{Tr} \mathbf{i}(\alpha, \beta, \gamma)$, $\left(G_{2}, w\right)$ is not extremal.

Proof. Suppose $\left(G_{2}, w\right)$ is extremal; by Lemma 4.6 we must have $t\left(G_{2}, w\right)<\alpha \beta+\gamma-1$. The edge and triangle densities are given by

$$
\begin{gathered}
\alpha=\hat{b}_{1}+\left(1-\hat{b}_{1}\right) \hat{c}_{1}, \\
\beta=1-\hat{a}_{1}+\hat{a}_{1} \hat{c}_{1}, \\
\gamma=\hat{a}_{1} \hat{b}_{1}+\left(1-\hat{a}_{1}\right)\left(1-\hat{b}_{1}\right), \\
t\left(G_{2}, w\right)=\hat{a}_{1} \hat{b}_{1} \hat{c}_{1}+\left(1-\hat{a}_{1}\right)\left(1-\hat{b}_{1}\right) \hat{c}_{1} .
\end{gathered}
$$

Substituting into $t\left(G_{2}, w\right)<\alpha \beta+\gamma-1$ and simplifying yields

$$
\hat{a}_{1}\left(1-\hat{b}_{1}\right)\left(1-\hat{c}_{1}\right)\left(1+\hat{c}_{1}\right)<0
$$

which is false.

Lemma 4.18. For $(\alpha, \beta, \gamma) \in R_{2}$ there exist no weightings $w$ of $G_{3}$ such that $\left(G_{3}, w\right) \in$ $\operatorname{Tr} \mathbf{i}(\alpha, \beta, \gamma)$. 
Proof. $G_{3}$ is strongly-isomorphic to $H_{6}$. Hence the result follows immediately from Lemma 3.4.

Lemma 4.19. If $(\alpha, \beta, \gamma) \in R_{2}$ then, for all weightings $w$ such that $\left(G_{4}, w\right) \in \operatorname{Tr} \mathbf{i}(\alpha, \beta, \gamma)$, $\left(G_{4}, w\right)$ is either not extremal or not vertex minimal.

Proof. Let us assume $\left(G_{4}, w\right) \in \operatorname{Tr} \mathbf{i}(\alpha, \beta, \gamma)$ is vertex minimal, and so $w(v) \neq 0$ for all $v \in V\left(G_{4}\right)$. By Lemma 3.3(ii) we also have $\alpha, \beta, \gamma \neq 0,1$. The densities in terms of the vertex weights $\hat{a}_{1}, \hat{b}_{2}, \hat{c}_{1}$, and $\hat{c}_{3}$, are as follows:

$$
\begin{gathered}
\alpha=1-\hat{b}_{2} \hat{c}_{1}, \\
\beta=1-\hat{a}_{1} \hat{c}_{3}, \\
\gamma=\hat{a}_{1} \hat{b}_{2}, \\
t\left(G_{4}, w\right)=\hat{a}_{1} \hat{b}_{2}\left(1-\hat{c}_{1}-\hat{c}_{3}\right) .
\end{gathered}
$$

We can use these equations to write $\hat{a}_{1}, \hat{b}_{2}, \hat{c}_{3}$, and $t\left(G_{4}, w\right)$ in terms of $\hat{c}_{1}$,

$$
\begin{gathered}
\hat{b}_{2}=\frac{1-\alpha}{\hat{c}_{1}} \\
\hat{a}_{1}=\frac{\gamma \hat{c}_{1}}{1-\alpha} \\
\hat{c}_{3}=\frac{(1-\alpha)(1-\beta)}{\gamma \hat{c}_{1}}, \\
t\left(G_{4}, w\right)=\gamma-\gamma \hat{c}_{1}-\frac{(1-\alpha)(1-\beta)}{\hat{c}_{1}} .
\end{gathered}
$$

From (4.15) we can deduce that $t\left(G_{4}, w\right)$ will be minimized when $\hat{c}_{1}$ is as large or as small as possible, because the second derivative with respect to $\hat{c}_{1}$ is negative. Since $\hat{b}_{2} \leqslant 1$ and $\hat{a}_{1} \leqslant 1,(4.13)$ and (4.14) imply that $\hat{c}_{1} \in[1-\alpha,(1-\alpha) / \gamma]$.

Substituting $\hat{c}_{1}=1-\alpha$ into (4.15) gives $t\left(G_{4}, w\right)=\alpha \gamma+\beta-1$. Substituting $\hat{c}_{1}=(1-\alpha) / \gamma$ into (4.15) gives $t\left(G_{4}, w\right)=\beta \gamma+\alpha-1$. Hence for $\hat{c}_{1} \in[1-\alpha,(1-\alpha) / \gamma]$ we have

$$
t\left(G_{4}, w\right) \geqslant \min \{\alpha \gamma+\beta-1, \beta \gamma+\alpha-1\} .
$$

Lemma 4.6 therefore tells us that $\left(G_{4}, w\right)$ can not be extremal.

Lemma 4.20. If $(\alpha, \beta, \gamma) \in R_{2}$ then, for all weightings $w$ such that $\left(G_{5}, w\right) \in \operatorname{Tri}(\alpha, \beta, \gamma)$, $\left(G_{5}, w\right)$ is either not extremal or not vertex minimal.

Proof. Suppose $(\alpha, \beta, \gamma) \in R_{2}$ and $\left(G_{5}, w\right) \in \operatorname{Tr} \mathbf{i}(\alpha, \beta, \gamma)$. We will show that there exists a weighting $w^{\prime}$ of $G_{4}$ such that $\left(G_{4}, w^{\prime}\right) \in \operatorname{Tr} i(\alpha, \beta, \gamma)$ and $t\left(G_{4}, w^{\prime}\right)=t\left(G_{5}, w\right)$. Since $\left|V\left(G_{4}\right)\right|=$ $\left|V\left(G_{5}\right)\right|$, Lemma 4.19 implies that $\left(G_{5}, w\right)$ is either not extremal or not vertex minimal.

Suppose $\left(G_{5}, w\right)$ is vertex minimal, in which case we may assume $w(v)>0$ for all $v \in V\left(G_{5}\right)$. To prove there exists $\left(G_{4}, w^{\prime}\right)$ with the same densities as $\left(G_{5}, w\right)$, note that $\Gamma_{B}\left(a_{1}\right)=\Gamma_{B}\left(a_{2}\right)$ in $G_{5}$. Hence we can modify $G_{5}$ by applying Merge on $a_{1}, a_{2}$, labelling the resulting merged vertex by $a$. This creates one partial edge $a c_{2}$. Apply Split on this edge, to remove it, choosing to replace the vertex $c_{2}$. The resulting weighted tripartite graph has the same densities as $\left(G_{5}, w\right)$ and it is easy to check that it is strongly-isomorphic to $G_{4}$.

Lemma 4.21. If $(\alpha, \beta, \gamma) \in R_{2}$ then, for all weightings $w$ such that $\left(G_{6}, w\right) \in \operatorname{Tr} \mathbf{i}(\alpha, \beta, \gamma)$, $\left(G_{6}, w\right)$ is either not extremal or not vertex minimal.

Proof. Suppose $(\alpha, \beta, \gamma) \in R_{2}$ and $\left(G_{6}, w\right) \in \operatorname{Tr} i(\alpha, \beta, \gamma)$. We will show that there exists a weighting $w^{\prime}$ of $G_{5}$ such that $\left(G_{5}, w^{\prime}\right) \in \operatorname{Tr} i(\alpha, \beta, \gamma)$ and $t\left(G_{5}, w^{\prime}\right)=t\left(G_{6}, w\right)$. Since $\left|V\left(G_{5}\right)\right|=$ $\left|V\left(G_{6}\right)\right|$, Lemma 4.20 implies that $\left(G_{6}, w\right)$ is either not extremal or not vertex minimal. 
Suppose $\left(G_{6}, w\right)$ is vertex minimal, in which case we may assume $w(v)>0$ for all $v \in V\left(G_{6}\right)$. To prove there exists $\left(G_{5}, w^{\prime}\right)$ with the same densities as $\left(G_{6}, w\right)$, note that $\Gamma_{C}\left(b_{2}\right)=\Gamma_{C}\left(b_{3}\right)$ in $G_{6}$. Hence we can modify $G_{6}$ by applying Merge on $b_{2}, b_{3}$, labelling the resulting merged vertex $b$. This creates one partial edge $a_{2} b$. Apply Split on that edge, to remove it, choosing to replace the vertex $a_{2}$. The resulting weighted tripartite graph has the same densities as $\left(G_{6}, w\right)$ and it is easy to check that it is strongly-isomorphic to $G_{5}$.

Lemma 4.22. For $(\alpha, \beta, \gamma) \in R_{2}$ there exist no weightings $w$ of $G_{9}$ such that $\left(G_{9}, w\right) \in$ $\operatorname{Tr} \mathbf{i}(\alpha, \beta, \gamma)$.

Proof. Suppose $\left(G_{9}, w\right) \in \operatorname{Tr} \mathbf{i}(\alpha, \beta, \gamma)$ for $(\alpha, \beta, \gamma) \in R_{2}$. If $w\left(c_{2}\right)=0$ then removing $c_{2}$ leaves $G_{9}$ strongly-isomorphic to $H_{6}$. Hence we get a contradiction from Lemma 3.4. If $w\left(c_{1}\right)=0$ or $w\left(b_{2}\right)=0$ then $\alpha=1$, and $(1, \beta, \gamma) \notin R_{2}$ by Lemma 3.3(ii). Similarly we can show all other vertices must have a non-zero weight. We will get a contradiction by showing that $\Delta(\alpha, \beta, \gamma) \geqslant 0$ and hence $(\alpha, \beta, \gamma) \notin R_{2}$.

Consider a new weighting $w^{\prime}$ given by $w^{\prime}(v)=w(v)$ for all $v \in V\left(G_{9}\right) \backslash\left\{c_{1}, c_{2}\right\}, w^{\prime}\left(c_{1}\right)=$ $w\left(c_{1}\right)+w\left(c_{2}\right)$ and $w^{\prime}\left(c_{2}\right)=0$. For convenience let us write $\alpha^{\prime}=\alpha\left(G_{9}, w^{\prime}\right)$ (note that $\beta\left(G_{9}, w^{\prime}\right)=\beta$ and $\left.\gamma\left(G_{9}, w^{\prime}\right)=\gamma\right)$. Since $w^{\prime}\left(c_{2}\right)=0$ we could remove it from $G_{9}$ without changing any densities and the resulting weighted tripartite graph would be stronglyisomorphic to $H_{6}$; let $w^{\prime \prime}$ be the corresponding weighting. Since $w(v) \neq 0$ for all $v \in V\left(G_{9}\right)$, we know $w^{\prime \prime}(v) \neq 0$ for all $v \in V\left(H_{6}\right)$, and consequently $t\left(H_{6}, w^{\prime \prime}\right)>0$. Lemma 3.2 tells us that $T_{\min }\left(\alpha^{\prime}, \beta, \gamma\right)=t\left(H_{6}, w^{\prime \prime}\right)>0$; therefore by Theorem 2.2 we have $\left(\alpha^{\prime}, \beta, \gamma\right) \in R$. Moreover, Lemma 3.4 implies that $\Delta\left(\alpha^{\prime}, \beta, \gamma\right) \geqslant 0$.

Since $\alpha^{\prime}=1-w^{\prime}\left(b_{2}\right) w^{\prime}\left(c_{1}\right)=1-w\left(b_{2}\right) w\left(c_{1}\right)-w\left(b_{2}\right) w\left(c_{2}\right)$, we have

$$
\alpha^{\prime}=\alpha-w\left(b_{2}\right) w\left(c_{2}\right) \text {. }
$$

Hence we can write $\alpha=\alpha^{\prime}+\epsilon$, where $\epsilon=w\left(b_{2}\right) w\left(c_{2}\right)>0$. Consider

$$
\begin{aligned}
\Delta(\alpha, \beta, \gamma) & =\Delta\left(\alpha^{\prime}+\epsilon, \beta, \gamma\right) \\
& =\Delta\left(\alpha^{\prime}, \beta, \gamma\right)+2 \epsilon \alpha^{\prime}+\epsilon^{2}-2 \epsilon \beta-2 \epsilon \gamma+4 \epsilon \beta \gamma \\
& =\Delta\left(\alpha^{\prime}, \beta, \gamma\right)+\epsilon^{2}+2 \epsilon\left(\alpha^{\prime}+\beta+\gamma-2\right)+4 \epsilon(1-\beta)(1-\gamma) \\
& =\Delta\left(\alpha^{\prime}, \beta, \gamma\right)+\epsilon^{2}+2 \epsilon t\left(H_{6}, w^{\prime \prime}\right)+4 \epsilon(1-\beta)(1-\gamma) .
\end{aligned}
$$

Since each term is non-negative we have $\Delta(\alpha, \beta, \gamma) \geqslant 0$. Therefore $(\alpha, \beta, \gamma) \notin R_{2}$, which is a contradiction.

Lemma 4.23. For all weightings $w$ such that $\left(G_{10}, w\right) \in \operatorname{Tri},\left(G_{10}, w\right)$ is either not extremal or not vertex minimal.

Proof. Suppose $\left(G_{10}, w\right)$ is extremal and vertex minimal; hence $w(v) \neq 0$ for all $v \in V\left(G_{10}\right)$. Convert $\left(G_{10}, w\right)$ into a doubly-weighted tripartite graph by adding the function $p$ which maps all edges to 1 . Applying Merge on $\left(G_{10}, w, p\right)$ and $b_{2}, b_{3}$ results in only one partial edge being created $b c_{2}$ (where $b$ is the vertex replacing $b_{2}, b_{3}$ ). We can apply Split on that edge, choosing to replace the vertex $c_{2}$, and then revert back to a weighted graph $\left(G_{10}^{\prime}, w^{\prime}\right)$ say. Now $\left(G_{10}^{\prime}, w^{\prime}\right)$ has the same densities as $\left(G_{10}, w\right)$ but $G_{10}^{\prime}$ has $|B|=2$ and $|C|=3$. Moreover, $G_{10}$ has the property that $\Gamma_{B}\left(a_{1}\right)=\Gamma_{B}\left(a_{3}\right)$, and this is also true in $G_{10}^{\prime}$. Hence, applying Lemma 4.10 to $\left(G_{10}^{\prime}, w^{\prime}\right)$ and $a_{1}, a_{3}$, we see that $\left(G_{10}^{\prime}, w^{\prime}\right)$ is not extremal or not vertex minimal. Since $\left|V\left(G_{10}^{\prime}\right)\right|=\left|V\left(G_{10}\right)\right|$ the same is true of $\left(G_{10}, w\right)$.

Lemma 4.24. For all weightings $w$ such that $\left(G_{11}, w\right) \in \operatorname{Tri},\left(G_{11}, w\right)$ is either not extremal or not vertex minimal.

Proof. The proof is almost identical to that of Lemma 4.23. The only difference is at the end, where now we have $\Gamma_{B}\left(a_{1}\right)=\Gamma_{B}\left(a_{2}\right)$ holding true, and so we apply Lemma 4.10 to vertices $a_{1}$ and $a_{2}$ instead. 
Lemma 4.25. For all weightings $w$ such that $\left(G_{12}, w\right) \in \operatorname{Tri},\left(G_{12}, w\right)$ is either not extremal or not vertex minimal.

Proof. Suppose $\left(G_{12}, w\right)$ is vertex minimal, so $w(v)>0$ for all $v \in V\left(G_{12}\right)$. Of the three statements $\hat{a}_{1} \leqslant \hat{a}_{2}, \hat{b}_{1} \leqslant \hat{b}_{2}, \hat{c}_{1} \leqslant \hat{c}_{2}$, at least two must be true or at least two must be false. Without loss of generality, let us suppose that $\hat{a}_{1} \leqslant \hat{a}_{2}, \hat{b}_{1} \leqslant \hat{b}_{2}$ are both true.

The densities of $\left(G_{12}, w\right)$ are given by

$$
\begin{aligned}
t\left(G_{12}, w\right) & =\hat{a}_{3} \hat{b}_{3} \hat{c}_{3}, \\
\alpha & =\hat{b}_{1} \hat{c}_{2}+\hat{b}_{2} \hat{c}_{1}+\hat{c}_{3}, \\
\beta & =\hat{a}_{1} \hat{c}_{2}+\hat{a}_{2} \hat{c}_{1}+\hat{a}_{3}, \\
\gamma & =\hat{a}_{1} \hat{b}_{2}+\hat{a}_{2} \hat{b}_{1}+\hat{b}_{3} .
\end{aligned}
$$

Consider the doubly-weighted tripartite graph $\left(G_{12}, w, p\right)$ where $p$ maps all edges to 1 . It has the same densities as $\left(G_{12}, w\right)$. If we move a sufficiently small amount of weight $\delta>0$ from vertex $c_{2}$ to $c_{1}, \alpha$ and $\beta$ increase. By decreasing $p\left(b_{3} c_{3}\right)$ and $p\left(a_{3} c_{3}\right)$ respectively we can keep all densities unchanged. More precisely set

$$
p\left(b_{3} c_{3}\right)=1-\delta\left(\hat{b}_{2}-\hat{b}_{1}\right) / \hat{b}_{3} \hat{c}_{3}, \quad p\left(a_{3} c_{3}\right)=1-\delta\left(\hat{a}_{2}-\hat{a}_{1}\right) / \hat{a}_{3} \hat{c}_{3} .
$$

If $\hat{a}_{1}=\hat{a}_{2}$ and $\hat{b}_{1}=\hat{b}_{2}$, then increasing the weight of $c_{1}$ to $\hat{c}_{1}+\hat{c}_{2}$ and removing $c_{2}$ will result in a weighted tripartite graph with the same densities as $\left(G_{12}, w\right)$ but with fewer vertices. Hence we know that $p\left(b_{3} c_{3}\right)<1$ or $p\left(a_{3} c_{3}\right)<1$. Consequently we now have a doubly-weighted tripartite graph with the same edge densities as $\left(G_{12}, w\right)$ but a strictly smaller triangle density. Hence by Lemma 4.1, $\left(G_{12}, w\right)$ is not extremal.

Suppose now that two of the statements $\hat{a}_{1} \leqslant \hat{a}_{2}, \hat{b}_{1} \leqslant \hat{b}_{2}$ and $\hat{c}_{1} \leqslant \hat{c}_{2}$ are false; for example, $\hat{a}_{1}>\hat{a}_{2}$ and $\hat{b}_{1}>\hat{b}_{2}$. We can repeat the above argument, this time moving weight from $c_{1}$ to $c_{2}$, again constructing a doubly-weighted tripartite graph with the same edge densities but a smaller triangle density.

Lemma 4.26. If $(\alpha, \beta, \gamma) \in R_{2}$ then, for all weightings $w$ such that $\left(G_{14}, w\right) \in \operatorname{Tri}(\alpha, \beta, \gamma)$, $\left(G_{14}, w\right)$ is either not extremal or not vertex minimal.

Proof. Suppose $\left(G_{14}, w\right)$ is extremal and vertex minimal, so $w(v)>0$ for all $v \in V\left(G_{14}\right)$. Consider the doubly-weighted tripartite graph $\left(G_{14}, w, p\right)$, where $p$ maps all edges to 1 . Applying Lemma 4.3 to $\left(G_{14}, w, p\right)$ on the non-edge $a_{1} b_{1}$ and the edge $a_{3} b_{2}$ tells us that, in order to be extremal,

$$
\sum_{c \in C_{a_{1} b_{1}}} w(c) \geqslant \sum_{c \in C_{a_{3} b_{2}}} w(c)
$$

must hold. Since $C_{a_{1} b_{1}}=\left\{c_{2}, c_{3}\right\}$ and $C_{a_{3} b_{2}}=\left\{c_{1}\right\}$ we must have $\hat{c}_{2}+\hat{c}_{3} \geqslant \hat{c}_{1}$ or equivalently $1-2 \hat{c}_{1} \geqslant 0$ (using the fact that $\hat{c}_{1}+\hat{c}_{2}+\hat{c}_{3}=1$ ). Similarly we can show that $1-2 \hat{c}_{2} \geqslant 0$ by looking at $a_{2} b_{2}, a_{1} b_{3}$, and $1-2 \hat{c}_{3} \geqslant 0$ by taking $a_{3} b_{3}, a_{2} b_{1}$. By symmetry we must have $1-2 w(v) \geqslant 0$ for all $v \in V\left(G_{14}\right)$. Note that the function $w^{\prime}$ defined by $w^{\prime}(v)=1-2 w(v)$ for all $v \in V\left(G_{14}\right)$ provides a valid weighting of $G_{14}$, as $w^{\prime}(v) \geqslant 0$ for all $v \in V\left(G_{14}\right)$ and the sum of the weights in a class, $X$ say, is

$$
\begin{aligned}
\sum_{v \in X} w^{\prime}(v) & =\sum_{v \in X}(1-2 w(v)) \\
& =|X|-2 \sum_{v \in X} w(v) \\
& =|X|-2 \\
& =1
\end{aligned}
$$

because every class in $G_{14}$ has size 3 . 
Recall that $\bar{G}_{14}$ is the tripartite complement of the graph $G_{14}$. Consider the weighted tripartite graph $\left(\bar{G}_{14}, w^{\prime}\right)$, with edge densities

$$
\alpha\left(\bar{G}_{14}, w^{\prime}\right)=\alpha^{\prime}, \quad \beta\left(\bar{G}_{14}, w^{\prime}\right)=\beta^{\prime}, \quad \gamma\left(\bar{G}_{14}, w^{\prime}\right)=\gamma^{\prime} .
$$

We can write down $\alpha^{\prime}$ in terms of $\alpha$ :

$$
\begin{aligned}
\alpha^{\prime} & =\left(1-2 \hat{b}_{1}\right)\left(1-2 \hat{c}_{1}\right)+\left(1-2 \hat{b}_{2}\right)\left(1-2 \hat{c}_{2}\right)+\left(1-2 \hat{b}_{3}\right)\left(1-2 \hat{c}_{3}\right) \\
& =3-2\left(\hat{b}_{1}+\hat{b}_{2}+\hat{b}_{3}\right)-2\left(\hat{c}_{1}+\hat{c}_{2}+\hat{c}_{3}\right)+4\left(\hat{b}_{1} \hat{c}_{1}+\hat{b}_{2} \hat{c}_{2}+\hat{b}_{3} \hat{c}_{3}\right) \\
& =3-4\left(1-\hat{b}_{1} \hat{c}_{1}-\hat{b}_{2} \hat{c}_{2}-\hat{b}_{3} \hat{c}_{3}\right) \\
& =3-4 \alpha
\end{aligned}
$$

similarly $\beta^{\prime}=3-4 \beta$ and $\gamma^{\prime}=3-4 \gamma$. Next let us write $t\left(\bar{G}_{14}, w^{\prime}\right)$ in terms of $t\left(G_{14}, w\right)$ :

$$
\begin{aligned}
t\left(\bar{G}_{14}, w^{\prime}\right)= & \left(1-2 \hat{a}_{1}\right)\left(1-2 \hat{b}_{1}\right)\left(1-2 \hat{c}_{1}\right)+\left(1-2 \hat{a}_{2}\right)\left(1-2 \hat{b}_{2}\right)\left(1-2 \hat{c}_{2}\right) \\
& +\left(1-2 \hat{a}_{3}\right)\left(1-2 \hat{b}_{3}\right)\left(1-2 \hat{c}_{3}\right) \\
= & 3-2\left(\hat{a}_{1}+\hat{a}_{2}+\hat{a}_{3}\right)-2\left(\hat{b}_{1}+\hat{b}_{2}+\hat{b}_{3}\right)-2\left(\hat{c}_{1}+\hat{c}_{2}+\hat{c}_{3}\right) \\
& +4\left(\hat{a}_{1} \hat{b}_{1}+\hat{a}_{1} \hat{c}_{1}+\hat{b}_{1} \hat{c}_{1}+\hat{a}_{2} \hat{b}_{2}+\hat{a}_{2} \hat{c}_{2}+\hat{b}_{2} \hat{c}_{2}+\hat{a}_{3} \hat{b}_{3}+\hat{a}_{3} \hat{c}_{3}+\hat{b}_{3} \hat{c}_{3}\right) \\
& -8\left(\hat{a}_{1} \hat{b}_{1} \hat{c}_{1}+\hat{a}_{2} \hat{b}_{2} \hat{c}_{2}+\hat{a}_{3} \hat{b}_{3} \hat{c}_{3}\right) \\
= & 1+4\left(\hat{a}_{1} \hat{b}_{1}+\hat{a}_{1} \hat{c}_{1}+\hat{b}_{1} \hat{c}_{1}+\hat{a}_{2} \hat{b}_{2}+\hat{a}_{2} \hat{c}_{2}+\hat{b}_{2} \hat{c}_{2}+\hat{a}_{3} \hat{b}_{3}+\hat{a}_{3} \hat{c}_{3}+\hat{b}_{3} \hat{c}_{3}\right. \\
& \left.-2 \hat{a}_{1} \hat{b}_{1} \hat{c}_{1}-2 \hat{a}_{2} \hat{b}_{2} \hat{c}_{2}-2 \hat{a}_{3} \hat{b}_{3} \hat{c}_{3}-\hat{a}_{1}-\hat{a}_{2}-\hat{a}_{3}\right) \\
= & 1+4\left(\left(1-\hat{a}_{1}\right) \hat{b}_{1} \hat{c}_{1}+\left(1-\hat{a}_{2}\right) \hat{b}_{2} \hat{c}_{2}+\left(1-\hat{a}_{3}\right) \hat{b}_{3} \hat{c}_{3}\right. \\
& \left.-\hat{a}_{1}\left(1-\hat{b}_{1}\right)\left(1-\hat{c}_{1}\right)-\hat{a}_{2}\left(1-\hat{b}_{2}\right)\left(1-\hat{c}_{2}\right)-\hat{a}_{3}\left(1-\hat{b}_{3}\right)\left(1-\hat{c}_{3}\right)\right) \\
= & 1+4\left(\left(\hat{a}_{2}+\hat{a}_{3}\right) \hat{b}_{1} \hat{c}_{1}+\left(\hat{a}_{1}+\hat{a}_{3}\right) \hat{b}_{2} \hat{c}_{2}+\left(\hat{a}_{1}+\hat{a}_{2}\right) \hat{b}_{3} \hat{c}_{3}\right. \\
& \left.-\hat{a}_{1}\left(\hat{b}_{2}+\hat{b}_{3}\right)\left(\hat{c}_{2}+\hat{c}_{3}\right)-\hat{a}_{2}\left(\hat{b}_{1}+\hat{b}_{3}\right)\left(\hat{c}_{1}+\hat{c}_{3}\right)-\hat{a}_{3}\left(\hat{b}_{1}+\hat{b}_{2}\right)\left(\hat{c}_{1}+\hat{c}_{2}\right)\right) \\
= & 1+4\left(-\hat{a}_{1} \hat{b}_{2} \hat{c}_{3}-\hat{a}_{1} \hat{b}_{3} \hat{c}_{2}-\hat{a}_{2} \hat{b}_{1} \hat{c}_{3}-\hat{a}_{2} \hat{b}_{3} \hat{c}_{1}-\hat{a}_{3} \hat{b}_{1} \hat{c}_{2}-\hat{a}_{3} \hat{b}_{2} \hat{c}_{1}\right) \\
= & 1-4 t\left(G_{14}, w\right) .
\end{aligned}
$$

Without loss of generality, suppose $\alpha^{\prime} \leqslant \beta^{\prime} \leqslant \gamma^{\prime}$. Since $\left(G_{14}, w\right)$ is extremal by Lemma 4.5 we have

$$
t\left(G_{14}, w\right) \leqslant 2 \sqrt{\alpha \beta(1-\gamma)}+2 \gamma-2
$$

Rewriting in terms of $\alpha^{\prime}, \beta^{\prime}, \gamma^{\prime}, t\left(\bar{G}_{14}, w^{\prime}\right)$ gives

$$
3+2 \gamma^{\prime}-t\left(\bar{G}_{14}, w^{\prime}\right) \leqslant \sqrt{\left(3-\alpha^{\prime}\right)\left(3-\beta^{\prime}\right)\left(1+\gamma^{\prime}\right)} .
$$

Note that in any weighted tripartite graph the triangle density is bounded above by all of the edge densities, thus $t\left(\bar{G}_{14}, w^{\prime}\right) \leqslant \alpha^{\prime}$, and so

$$
3+2 \gamma^{\prime}-\alpha^{\prime} \leqslant \sqrt{\left(3-\alpha^{\prime}\right)\left(3-\beta^{\prime}\right)\left(1+\gamma^{\prime}\right)} .
$$

Squaring both sides and rearranging yields

$$
\alpha^{\prime 2}+\gamma^{\prime}\left(4 \gamma^{\prime}-\alpha^{\prime} \beta^{\prime}\right)+\gamma^{\prime}\left(3 \beta^{\prime}-\alpha^{\prime}\right)+3\left(\gamma^{\prime}-\alpha^{\prime}\right)+\beta^{\prime}\left(3-\alpha^{\prime}\right) \leqslant 0 .
$$

Each term is non-negative (because $0 \leqslant \alpha^{\prime} \leqslant \beta^{\prime} \leqslant \gamma^{\prime} \leqslant 1$ ), and so the only way this can be true is if $\alpha^{\prime}=\beta^{\prime}=\gamma^{\prime}=0$. Hence $\alpha=\beta=\gamma=3 / 4$, but such values do not lie in $R_{2}$ due to the fact that $\Delta(3 / 4,3 / 4,3 / 4)=0$. Thus we have a contradiction and our assumption that $\left(G_{14}, w\right)$ is extremal and vertex minimal must be false.

Proof of Theorem 2.5. Our computer search tells us that the only possible extremal and vertex minimal tripartite graphs are strongly-isomorphic to those given in Figure 11. Given $(\alpha, \beta, \gamma) \in R_{2}$ for all weightings $w$, then $\left(G_{1}, w\right),\left(G_{2}, w\right),\left(G_{3}, w\right),\left(G_{4}, w\right),\left(G_{5}, w\right),\left(G_{6}, w\right)$, 
$\left(G_{9}, w\right),\left(G_{10}, w\right),\left(G_{11}, w\right),\left(G_{12}, w\right),\left(G_{14}, w\right)$ are either not extremal, not vertex minimal or do not lie in $\operatorname{Tri}(\alpha, \beta, \gamma)$ by Lemmas $4.16-4.26$, respectively. This just leaves $G_{7}, G_{8}$ and $G_{13}$ which are strongly-isomorphic to $H_{7}^{\prime}, H_{7}$ and $H_{9}$, respectively.

\section{Conjectures}

The following conjecture, if true, would allow us to write $T_{\min }(\alpha, \beta, \gamma)$ as a simple expression for all values of $\alpha, \beta, \gamma \in[0,1]$.

Conjecture 5.1. For $\gamma \leqslant \alpha, \beta$,

$$
T_{\min }(\alpha, \beta, \gamma)= \begin{cases}0 & \text { if }(\alpha, \beta, \gamma) \in[0,1]^{3} \backslash R \\ 2 \sqrt{\alpha \beta(1-\gamma)}+2 \gamma-2 & \text { if }(\alpha, \beta, \gamma) \in R_{2} \\ \alpha+\beta+\gamma-2 & \text { otherwise }\end{cases}
$$

To prove Conjecture 5.1 it is sufficient to prove the subsequent conjecture.

Conjecture 5.2. If $(\alpha, \beta, \gamma) \in R_{2}$ then, for all weightings $w$ such that $\left(H_{9}, w\right) \in$ $\operatorname{Tr} \mathbf{i}(\alpha, \beta, \gamma),\left(H_{9}, w\right)$ is either not extremal or not vertex minimal.

\section{Theorem 5.3. Conjecture 5.2 implies Conjecture 5.1.}

Proof. Theorems 2.2 and 2.4 tell us when $T_{\min }(\alpha, \beta, \gamma)=0$ and $\alpha+\beta+\gamma-2$, respectively. By Theorem 2.5 and Conjecture 5.2 we know that the only extremal tripartite graphs we have to consider are $H_{7}$ and $H_{7}^{\prime}$. Let us show that $H_{7}^{\prime}$ can do no better than $H_{7}$.

Let $(\alpha, \beta, \gamma) \in R_{2}$ and $\left(H_{7}^{\prime}, w^{\prime}\right) \in \operatorname{Tr} \mathbf{T}(\alpha, \beta, \gamma)$. We need to show there exists a weighting $w$ for $H_{7}$ so that $\left(H_{7}, w\right)$ has the same densities as $\left(H_{7}^{\prime}, w^{\prime}\right)$. Note that $\Gamma_{A}\left(b_{2}\right)=\Gamma_{A}\left(b_{3}\right)$ in $H_{7}^{\prime}$ and $w^{\prime}\left(b_{2}\right)+w^{\prime}\left(b_{3}\right)>0$ (otherwise $\alpha=1$ which can not occur according to Lemma 3.3(ii)). Hence we can modify $H_{7}^{\prime}$ by applying Merge on $b_{2}, b_{3}$, labelling the resulting merged vertex $b$. This creates one partial edge $b c_{2}$. Apply Split to this edge to remove it, choosing to replace the vertex $c_{2}$. The resulting weighted tripartite graph has the same densities as $\left(H_{7}^{\prime}, w^{\prime}\right)$ and it is easy to check that it is strongly-isomorphic to $H_{7}$.

Therefore when $(\alpha, \beta, \gamma) \in R_{2}$ we need only consider graphs strongly-isomorphic to $H_{7}$, and by Lemma 4.5 we get $T_{\min }(\alpha, \beta, \gamma)$ is equal to

$$
\min \{2 \sqrt{\alpha \beta(1-\gamma)}+2 \gamma-2,2 \sqrt{\alpha \gamma(1-\beta)}+2 \beta-2,2 \sqrt{\beta \gamma(1-\alpha)}+2 \alpha-2\} .
$$

To finish the proof let us show that $\gamma \leqslant \beta$ if and only if

$$
2 \sqrt{\alpha \gamma(1-\beta)}+2 \beta-2 \geqslant 2 \sqrt{\alpha \beta(1-\gamma)}+2 \gamma-2 .
$$

We can prove a similar result for $\gamma \leqslant \alpha$. For ease of notation let $d_{1}=2 \sqrt{\alpha \gamma(1-\beta)}+2 \beta-2$ and $d_{2}=\alpha+\beta+\gamma-2$. So we have

$$
\begin{array}{rlrl}
d_{1} & \geqslant 2 \sqrt{\alpha \beta(1-\gamma)}+2 \gamma-2 \\
& \Longleftrightarrow & \Longleftrightarrow & \\
d_{1}+2(1-\gamma) & \geqslant 2 \sqrt{\alpha \beta(1-\gamma)} \\
\left(d_{1}+2(1-\gamma)\right)^{2} & \geqslant 4 \alpha \beta(1-\gamma) \\
& =\left(d_{2}+2(1-\gamma)\right)^{2}-\Delta(\alpha, \beta, \gamma) \\
& & d_{1}^{2}+4 d_{1}(1-\gamma) & \geqslant d_{2}^{2}+4 d_{2}(1-\gamma)-\Delta(\alpha, \beta, \gamma) \\
& \Longleftrightarrow \quad d_{1}^{2}+4 d_{1}-d_{2}^{2}-4 d_{2}+\Delta(\alpha, \beta, \gamma) & \geqslant 4 \gamma\left(d_{1}-d_{2}\right) .
\end{array}
$$


By Lemma 3.1 we know $d_{1}-d_{2} \geqslant 0$. It is easy to check that $d_{1}-d_{2}=0$ implies $\Delta(\alpha, \beta, \gamma) \geqslant 0$ which is not true, since $(\alpha, \beta, \gamma) \in R_{2}$. Consequently we have

$$
d_{1} \geqslant 2 \sqrt{\alpha \beta(1-\gamma)}+2 \gamma-2 \Longleftrightarrow \frac{d_{1}^{2}+4 d_{1}-d_{2}^{2}-4 d_{2}+\Delta(\alpha, \beta, \gamma)}{4\left(d_{1}-d_{2}\right)} \geqslant \gamma
$$

Substituting $d_{1}=2 \sqrt{\alpha \gamma(1-\beta)}+2 \beta-2$ and $d_{2}=\alpha+\beta+\gamma-2$ into

$$
\frac{d_{1}^{2}+4 d_{1}-d_{2}^{2}-4 d_{2}+\Delta(\alpha, \beta, \gamma)}{4\left(d_{1}-d_{2}\right)}
$$

shows that it simplifies to $\beta$. Thus

$$
2 \sqrt{\alpha \gamma(1-\beta)}+2 \beta-2 \geqslant 2 \sqrt{\alpha \beta(1-\gamma)}+2 \gamma-2 \Longleftrightarrow \beta \geqslant \gamma
$$

\section{Appendix}

A $\mathrm{C}++$ implementation of the algorithm to produce the final list of extremal vertex minimal tripartite graphs may be downloaded from http://www.ucl.ac.uk/ ucahjmt/GraphFinder.cc. The graphs it outputs are given in Figure 11.

\section{References}

1. B. BollobÁs, 'Relations between sets of complete subgraphs', Proceedings of the Fifth British Combinatorial Conference, Congressus Numerantium 15 (eds C. St. J. A. Nash-Williams and J. Sheehan; Utilitas Mathematica, Winnipeg, 1976) 79-84.

2. A. Bondy, J. Shen, S. Thomassé and C. Thomassen, 'Density conditions for triangles in multipartite graphs', Combinatorica 26 (2006) no. 2, 121-131.

3. P. ERdős, 'On a theorem of Rademacher-Turán', Illinois J. Math. 6 (1962) 122-127.

4. D. C. Fisher, 'Lower bounds on the number of triangles in a graph', J. Graph Theory 13 (1989) no. 4, $505-512$.

5. L. LovÁsz and M. Simonovits, 'On the number of complete subgraphs of a graph, II', Studies in pure mathematics (Birkhäuser, Basel, 1983) 459-495.

6. V. W. Mantel, 'Problem 28', Wiskundige Opgaven 10 (1907) 60-61.

7. A. A. Razborov, 'On the minimal density of triangles in graphs', Combin. Probab. Comput. 17 (2008) no. $4,603-618$.

Rahil Baber

Department of Mathematics

UCL, Gower Street, London

WC1E $6 B T$

United Kingdom

rahilbaber@hotmail.com

John Talbot

Department of Mathematics

UCL, Gower Street, London

WC1E $6 B T$

United Kingdom

talbot@math.ucl.ac.uk
J. Robert Johnson

School of Mathematical Sciences

Queen Mary University of London

E1 4NS

United Kingdom

r.johnson@qmul.ac.uk 